Article

\title{
Floristic Study of Karnaphuli Range in Kaptai Reserve Forest, Rangamati, Bangladesh ${ }^{\dagger}$
}

\author{
Md. Rishad Abdullah *, AKM Golam Sarwar, Md. Ashrafuzzaman and Md. Mustafizur Rahman \\ Department of Crop Botany, Bangladesh Agricultural University, Mymensigh, Bangladesh; \\ drsarwar@bau.edu.bd; drashraf2007@yahoo.com; chairman.pcrf@gmail.com \\ * Correspondence: rishad.abdullah@gmail.com; Tel: +8801712894181 \\ + Location of study: Karnaphuli range in Kaptai Reserve forest, Rangamati, Bangladesh
}

\begin{abstract}
A botnical survey was conducted in Kaptai reserve forests under Rangamati district in Bangladesh to study the flora of Karnaphuli range from May 2015 to October 2018. The survey was accompanied by a collection of voucher specimens enumerates 464 plant species belonging to 334 genera under 117 families from the forest range. The survey has confirmed 31 threatened forest species from this area along with many near threatened plant species.
\end{abstract}

Keywords: flora; vascular plants; reserve forest; threatened plants; Kaptai

\section{Introduction}

Bangladesh is a small country, enriched with high plant diversity, since it lies in a transition of two mega-biodiversity hotspots, viz, Indo-Himalayas and Indo-Chinese (Nishat et al., 2002; Khan, 2003). Near about 5,700 species of angiosperms with, 3 species of gymnosperms, 29 orchids 68 woody legumes, 130 fibers yielding plants, 500 medicinal plants and 1,700 pteridophytes have been recorded so far (Islam, 2003). However, the database regarding the biodiversity in Bangladesh is not so rich and evidence is often based on secondary information or guessed works only. Government commitment is to survey their natural living resources, both domesticated and wild and to conserve site noted for their rich biological diversity as well as threatened species and domesticated varieties which is one of the essential elements of the Convention on Biological Diversity (Freedman, 1995), but identification of the various important components of biodiversity through systematic and scientific approach is still under develop in Bangladesh. Quantitative floristic inventories are fundamental to an understanding of the ecology of tropical forests and for developing rational forest management strategies (Campbell et al., 1986). Floristic studies help us to the assessment of the plant wealth and its potentiality of an area can understand by floral studies. It also helps us to know the basic facets of biology such as speciation, isolation, endemism and evolution. The floristic components of an area change from time to time, due to various ecological factors, mostly biotic (Rao et al., 2013).

Karnaphuli range of the Kaptai reserve forest under the Rangamati South Forest Division management. It is divided into two Kaptai and Karnaphuli ranges. Many floristic studies conducted here such as Heinig (1925) recorded 45 taxa, Uddin et al. (1998) 332 species; Nath et al. (1998) 85 tree species; Rashid and Chowdhury (2013) 43 taxa; Chowdhury et al. (2017) 86 tree species from Sitapahar, while Uddin and Hassan (2012) reported 41 pteridophyte species from Rampahar and Sitapahar reserve forests. From Rampahar, Uddin and Hassan (2012) reported 89 monocots, Feroz et al. (2014) 40 species and Rahman et al. (2016) 43 tree species and Chowdhury et al. (2018) 50 tree. Apart from these 2 areas of Sitapahar and Rampahr Mohajn et al. (2016) recorded 69 tree species Kaptai deer breeding centre of Karnaphuli range. Though the area is very rich in species diversity, a comprehensive floristic study of the area is still deficient. Our objective is to develop baseline information of the flora of Karnaphuli range of the Kaptai reserve forest which will eventually helpful for sustainable conservation and management activities by policymakers. 


\section{Materials and Methods}

The present study was carried out based on 27 field-investigation results from May 2015 to October 2018. Study area location confined in Karnphuli Sadar beat, Khalmukh beat of Karnaphuli range. During the field investigations, only those plants with reproductive organs such as flowers, fruits, and spores were collected as voucher specimens to maintain accuracy, and the collected specimens were stored at the Arshad Ali Khan Herbarium of Botanical garden in Bangladesh Agricultural University, Mymensingh, Bangladesh. The herbarium specimens were examined for comparison at the Bangladesh National Herbarium, Dhaka University Salar Khan Herbarium and Bangladesh Forest Research Institute Herbarium. The occurrences of the plants noted in the forest were compared with accounts gives in Hooker (1872-1897). Heining (1925), Uddin et al. (1998), Alam (1988), Ahmed et al. (2008), Khan et al (2001). Threatened plants were categorized with the help of Khan et al. (2001), Ahmed et al. (2008) and Ara et al. (2013). For nomenclature databases of The Plant List (2013) and Pasha and Uddin (2013) were also consulted.

\section{Results}

\section{Floristic composition of Karnaphuli Range Forest}

The vascular plants of Karnaphuli range found in this investigation included 463 species, 333 genera 117 families, one subspecies, 8 varieties. Among these total 378 species with 5 varieties under 89 families and 274 genera belonging to dicotyledons while a total of 72 species with 3 varieties under 17 families and 47 genera belonging to monocotyledons. Pteridophyta consists of 13 species under 11 families and 12 genera (Table 1, Annex 1\&2).

\section{Tree}

The study area was taxonomically well-represented with 140 tree species under 49 families and 112 genera. Moraceae had the maximum number of species (10 species) followed by Euphorbiaceae (9 species), Rutaceae and Anacardiaceae (7 species each); Fabaceae, Meliaceae, Myrtaceae, and Phyllanthaceae (6 species each), Caesalpiniaceae, Combretaceae and Lauraceae (5 species each); Bignoniaceae and Verbenaceae (4 species each); Annonoaceae, Burseraceae, Dipterocarpaceae, Ebenaceae, Flacourtiaceae, Myristicaceae, Rubiaceae, Sapindaceae, and Sterculiaceae (3 species each); Apocynaceae, Araliaceae, Dilleniaceae, Fagaceae, Mimosaceae, Oxalidaceae and Sapotaceae had two species each and 21 families with single species. Among genera Ficus and Syzygium (5 species each); Terminalia (4 species) were most dominant, followed by Dipterocarpus, Diospyros, Artocarpus and Cinnamomum (3 genera, each); Mangifera, Stereospermum, Dillenia, Albizia, Castanopsis, Averrhoa, Antidesma, Citrus, Clausena and Aporosa, had 2 species each and rest 94 genera with single species each. In the tree layer of the investigated area, Tectona grandis L.f., Lagerstroemia speciosa (L.) Pers., Dipterocarpus turbinatus Gaertn., Swietenia mahagoni (L.) Jacq. and Gmelina arborea Roxb. were dominant tree species used as main plantation tree species. Other common species were Syzygium fruticosum (Roxb.) DC., Palaquium polyanthum (Wall. ex G. Don.) Baill, Aphanamixis polystachya (Wall.) R.N.Parker, Baccaurea ramiflora Lour, Macaranga denticulata (Blume.) Muell. Arg., Bischofia javanica Blume., Protium serratum (Wall. ex Coelbr.) Engl etc (Annex 2).

\section{Shrubs}

A total of 98 shrubs species under 76 genera and 36 families were recorded from Karnaphuli Range Forest. Rubiaceae (9 species) the highest contributing family followed by Fabaceae (7 species); Lamiaceae and Verbenaceae (6 species each); Apocynaceae and Euphorbiaceae (5 species each); Leeaceae, Malvaceae and Urticaceae (4 species each); Acanthaceae, Asteraceae, Caesalpiniaceae, Melastomataceae, Myrsinaceae, Oleaceae and Rutaceae (3 species each); Annonaceae, Connaraceae, Lythraceae, Solanaceae, Sterculiaceae, Taccaceae and Tiliaceae (2 species each) and 13 families with single species. Among genera Clerodendrum, Leea and Ixora (4 species) 
were the most dominant species followed by Dracaena, Tabernaemontana, Jatropha, Flemingia, Senna, Uraria, Ocimum, Maesa, Jasminum, Mussaenda, Solanum, Tacca and Premna had each of 2 species while 60 genera with a single species each. The most common shrubs were Chromolaena odorata L., Tetracera sarmentosa (L.) Vahl., Microcos paniculata L., Cnesmone javanica Bl., Lantana camera L., Clerodendrum viscosum Vent., Rhyncotechum ellipticum (Wall ex D. Dietr.) A. DC., Mussaenda roxburghii Hook.f., Clerodendrum wallichii Merr., Murraya koenigii (L.) Spreng etc (Annex 2).

\section{Climber}

From our study, we recorded 63 climbers with 42 genera from 24 families. The most contributing families were Cucurbitaceae and Vitaceae (10 species each) followed by Menispermaceae (6 species); Dioscoreaceae (5 species); Piperaceae (4 species); Fabaceae (3 species) and Acanthaceae, Apocynaceae, Araceae, Rubiaceae, Convolvulaceae, Moraceae and Ascelpiadaceae, had 2 species individually, while the number of families with single species was 12 . In case of genera Cissus (6 species) and Dioscorea (5 species) were the most dominant followed by Momordica and Piper (4 species each); Cayratia (3 species); Tinospora, Curcuma and Stephania (2 species each) while the number of genera with single species was 34 . The most common climbers were Mikania cordata (Burm. f.) B.L.Rob. Thunbergia alata Bojer ex sims, Cissus adnata Roxb., Piper rhytidocarpum Hook. f. etc (Annex 2).

\section{Herbs}

144 herbs with 47 families and 109 genera were recorded from the Karnaphuli range. Zingiberaceae was the most contributing families with 20 species followed by Lamiaceae (11 species); Araceae (10 species); Asteraceae (7 species); Cyperaceae, Amaranthaceae and Fabaceae (6 species each); Urticaceae and Rubiaceae (5 species each); Malvaceae, Commelinaceae, Euphorbiaceae and Poaceae (4 species each); Liliaceae, Apiaceae, Orchidaceae, Convolvulaceae and Begoniaceae (3 species each); Solanaceae, Marantaceae, Polygonaceae, Acanthaceae, Linderniaceae, Gesneriaceae, Piperaceae and Caesalpiniaceae had 2 species each. Besides these 21 families were with single species each. Among genera Zingiber was the most contributing one with 5 species followed by Alpinia and Amaranthus (4 species each); Alocasia, Begonia, Curcuma, Desmodium, Kamepferia, and Leucas (3 species each); Commelina, Crotalaria, Cyperus, Hedychium, Hedyotis, Ipomoea, Lindernia, Ophiorrhiza, Peperomia, Pouzolzia, Senna, Sida and Stauranthera (2 species each), and 87 genera having single species. The most common species were Euphorbia hirta L., Amischotolype hookeri Hassk. H. Hara, Alpinia malaccensis Brum.f., Ocimum americum L., Typhonium trilobatum L., Portulaca oleracea L., Fleurya interrupta (L.) Gaudich and Alocasia fornicata (Roxb.) Schott. Etc (Annex 2).

\section{Epiphytes}

A total of 11 epiphytes were recorded in the study area. Among them, 5 were epiphytes belonging to 2 families and 5 genera from the angiosperm group and 6 species belonging to 6 genera and 6 families from Pteridophyta (Annex 1).

\section{Ferns}

The study recorded 7 fern species belonging to 6 genera and 7 families form the Karnaphuli range. The most common ferns were Pteris vittata L. and Dryopteris filixmas (L.) Schott etc (Annex 1).

\section{Threatened species}

The occurrence of 31 threatened plant species were confiremed in KFR during the survey. Among them 24 were vulnerable and 7 were endangered. Besides these 32 species were recorded as near threatened (Table 2). Among 7 endangered species one from shrubs (Pilea melastomoides (Poir.) Wedd.), two from climbers (Cucumis hystrix Chakrav and Myriopteron extensum (Wright \& Arn.) K. 
Schums.) One from epiphyte (Dischidia nummularia R. Br.), others belong to herbaceous plants (Hedyotis thomsonii Hook. f., Crepidium biauritum (Lindl.) D.L. and Globba pendula Roxb.). Among the vulnerable plants, 12 were tree species (Bhesa robusta (Roxb.) Ding Hou, Hopea odorata Roxb., Horsfieldia kingii (Hook. f.) Warb., Licuala peltata Roxb., Xerospermum laevigatum Radlk., Scaphium scaphigerum, Symplocos macrophylla Wall. ex DC ssp. Macrophylla, Mangifera sylvatica Roxb., Diospyros ramiflora Roxb., Terminalia chebula (Gaertn.) Retz., Terminalia arjuna (Roxb. ex DC.) Wight \& Arn and Glochidion zeylanicum (Gaertn.) A. Juss.); 4 were from shrubs (Harpullia arborea (Blanco) Radlk., Marsdenia tenacissima (Roxb.) Moon, Mycetia longifolia (Wall.) Kuntz. and Ixora spectabilis Wall. Ex G. Don) and 4 climbers (Rhaphidophora glauca (Wallich) Schott, Stixis suaveolens (Roxburgh) Pierre, Bull., Willughbeia edulis Roxb. and Dioscorea glabra Roxb.) (Annex 1\& 2).

\section{Discussion}

The present study results indicating that Karnaphuli forest range harbor of many plant species. Uddin et al. (1998) recorded 423 species belonging to 292 genera under 93 families from Sita Pahar at Kaptai reserve forest. Nath et al. (2000) also reported 85 tree species having $>10 \mathrm{~cm}$ dbh belonging to 36 families and 68 genera from the same reserve forest. Dutta et al. (2014) reported 332 vascular plant species belonging to 266 genera and 93 families from Sitakunda Botanical Garden and EcoPark. Uddin et al. (2013) recorded 535 angiosperms under 370 genera and 103 families from Teknaf Wildlife Sanctuary. It's diversity comparatively lower than Chunati Wildlife Sanctuary. Hossain and Hossain (2014) reported 691 plant species containing 240 trees, 102 shrubs, 211 herbs, 106 climbers, 19 ferns, 6 parasites and 7 epiphytes from Chunati Wildlife Sanctuary.

The present study revealed a total of 31 species threatened plant species which were more than Dutta et al. (2014) and Uddin and Hasan (2010). Dutta et al. (2014) recorded 29 rare and endangered species from Sitakunda Botanical Garden and Eco-Park while Uddin and Hasan (2010) recorded 19 threatened plant species from the Lawachara National Park, Bangladesh. Considering the results of these similar studies from different forest areas of Bangladesh, we can conclude that the Karnaphuli Range of Kaptai Reserve forest possesses comparatively well-diversified flora.

Acknowledgments: This study was carried out as a part of the research project, "Strengthening post graduate studies on plant genetic resource collection, conservation and characterization" (project number: CP-3021, 2014) supported by the University Grant Commission. The author's special thanks to the authority of the Bangladesh Forest Department for their co-operation during the field works and also grateful to the authorities of viz. Bangladesh National Herbarium (DACB), Dhaka University Salar Khan Herbarium and Bangladesh Forest Research Institute Herbarium, Chittagong (BFRI). for allowing consulting their libraries and using their herbarium materials.

\section{References}

Ahmed, Z.U., Begum, Z.N.T., Hassan, M.A., Khondker, M., Kabir, S.M.H., Ahmed, M., Ahmed, A.T.A., Rahman, A.K.T. and Haque, E.U. (Eds) 2008-2009. Encyclopedia of Flora and Fauna of Bangladesh, Vols. 6-10, 11, 12. Asiatic Society of Bangladesh, Dhaka.

Alam, M.K. 1988. Annotated checklist of woody flora of Sylhet forests. Bulletinn 5, Plant Taxonomy Series. Bangladesh Forest Research Institute, Chittagong, pp. 1-153.

Ara, H., Khan, B. and Uddin, S.N. (Eds) 2013. Red Data Book of Vascular Plants of Bangladesh. Bangladesh National Herbarium, Dhaka, pp. 1-280.

Campbell, D.D., Daly, D.C., Prance, G.T. and Maciel, U.N. 1986. Quantitative ecological inventory ofterra firm and varzea tropical forest on the Rio Xingu, Brazilian, Amazon. Brittonia 38: 369-393. https://doi.org/10.2307/2807085

Chowdhury, B., Hossain, M.K., Hossain, M.A. and Khan, B.M. 2018a: Native tree species diversity of Rampahar Natural Forest Reserve in Rangamati South Forest Division, Bangladesh. Ceylon Journal of Science 47(2) 129-136.

Chowdhury, P., Hossain, M.K., Hossain, M.A., Dutta, S. and Ray, T.K. 2017: Status, Wood Properties and Probable Uses of Lesser Used Species Recorded from Sitapahar Reserve Forest of Bangladesh. Indian For. 143(12):1241-1248. 
Dutta, S., Hossain, M.K., Hossain, M.A. and Chowdhury, P. 2014. Floral diversity of Sitakunda botanical garden and eco-park in Chittagong, Bangladesh. Indian J. Trop. Bio. 22:106-118.

Feroz, S.M., Alam, M.R., Das, P. and Al Mamun, A. 2014. Community ecology and spatial distribution of trees in a tropical wet evergreen forest in Kaptai national park in Chittagong Hill Tracts, Bangladesh. J. For. Res. 25(2):311-318.

Freedman, B. 1995. Environmental ecology. Second edition. Academic Press, New York, New York, USA.

Harun-Ur-Rashid, M. and Chowdhury, M.A.I 2013. Additions to the angiosperm flora in the Sitapahar Reserve Forest of Kaptai, Rangamati, Bangladesh. Bangladesh J. Plant Taxon. 20(2) 255-257.

Heinig RL. 1925. List of Plants of Chittagong Collectorate and the Hill Tracts. The Bengal Government Branch Press, Darjeeling, India. pp. 84.

Hooker, J.D. 1872-1897. The Flora of British India. Vols. 1-7. L. Reeve \& Co., Ashford, Kent, UK.

Hossain, M.K. and Hossain. M.A. 2014. Biodiversity of Chunati Wildlife Sanctuary: Flora. Arannayk Foundation and Bangladesh Forest Department. Dhaka, Bangladesh. pp. v + 175 .

Islam, S.S. 2003. State of forest genetic resources conservation and management in Bangladesh. Forest Genetic Resources Working Papers, Working Paper FGR/68E. Forest Resources Development Service, Forest Resources Division. FAO, Rome, Italy. 31pp.

Khan, M.S. 2003. Flora. In: Islam, S., Miah, S., Ahmed, W., Chowdhury, A. M., Rahman, S. M. M., Siddiqui, K. and Kabir, S. M. H. (eds), Banglapedia: National Encyclopaedia of Bangladesh. Vol. 4: 171-177. Asiatic Society of Bangladesh, Dhaka

Khan, M.S., Rahman, M.M. and Ali, M.A. (Eds) 2001. Red Data Book of Vascular Plants of Bangladesh, Vol. 1. Bangladesh National Herbarium, Dhaka, Bangladesh, pp. 1-179.

Mohajan, B., Hossain, M.K., Mahmud, A.A. and Khan, B.M. 2016: Diversity and structural composition of indigenous tree species at Kaptai deer breeding centre of Rangamati south forest division, Bangladesh. J. biodivers. conserv. bioresour. manag. 2(2):1-10.

Nath, T.K., Hossain, M.K. and Alam, M.K. 2000. Assessment of tree species diversity of Sitapahar forest reserve, Chittagong Hill Tracts (South) Forest Division, Bangladesh. Indian For. 126:16-21.

Nishat, A., Huq, S.M.I., Barua, S.P., Reza, A.H.M.A. and Khan, A.S.M. 2002. Bioecological Zones of Bangladesh. Dhaka: The World Conservation Union (IUCN) Country Office, Bangladesh, pp 33-101.

Pasha, M.K and Uddin, S.B. 2013. Dictionary of Plant Names of Bangladesh (Vascular Plants). Janokalyan Prokashani, Chittagong, pp. 1-320.

Rahman, M.M., Abdullah, M.R., Haque, M.E. and Fakir, M.S.A. 2016: Threatened plant species in protected areas in Bangladesh: Problems and prospects of conservation and utilization. 3rd National Conference on Natural Science and Technology; Asian University for Women, Chittagong, Bangladesh.

Rao, V.H., Gohil, T.G. and Thakor, A.B. 2013. Floristic study of Kaprada's hilly forest in south Gujarat. Int. J. Plant Sci. 7: 100-102.

The Plant List, 2013.The Plant List, a working list of all plant species. Version $1.1<\mathrm{http}$ ://www.theplantlist.org/>. Accessed on 29 October 2017.

Uddin, M.Z., Alam, F., Rhaman, A. and Hassan, A. 2013. Diversity in angiosperm flora of teknaf wildlife sanctuary, Bangladesh. Bangladesh J. Plant Taxon. 20: 145-162. https://doi.org/10.3329/bjpt.v20i2.17389

Uddin, S.N. and Hassan, M.A. 2012a: Pteridophyte flora of Rampahar and Sitapahar reserve forests under Rangamati district in Bangladesh. Dhaka Univ. J. Biol. Sci. 21(2): 153-161.

Uddin, S.N. and Hassan, M.A. 2012b: Angiosperm flora of Rampahar reserve forest under Rangamati district in Bangladesh. I. Liliopsida (Monocots). Bangladesh J. Plant Taxon. 19(1) 37-44.

Uddin, S.N., Khan, M.S., Hassan, M.A. and Alam, M.H. 1998. An annotated checklist of Angiosperm flora of Sitapahar at Kaptai in Bangladesh. Bangladesh J. Plant Taxon. 5: 13-46.

Uddin, M.Z. and Hassan, M.A. 2010. Angiosperm diversity of Lawachara National Park (Bangladesh): A preliminary assessment. Bangladesh J. Plant Taxon. 17(1): 9-22.

Table 1. Summary of the floristic of Karnaphuli range, Kaptai.

\begin{tabular}{ccccccc}
\hline Taxa & Family & Genera & Species & Subspecies & Variety & Total \\
\hline Pteridophyta & 11 & 12 & 13 & 0 & 0 & 13 \\
\hline Dicotyledons & 89 & 274 & 373 & 1 & 5 & 378 \\
Monocotyledons & 17 & 47 & 69 & 0 & 3 & 72 \\
\hline Total & 117 & 333 & 455 & 1 & 8 & 463 \\
\hline
\end{tabular}


Table 2. Conservation status of the collected species.

\begin{tabular}{|c|c|c|c|c|c|c|c|c|}
\hline \multirow{2}{*}{ Taxa } & \multirow{2}{*}{ Life form } & \multicolumn{6}{|c|}{ Conservation status } & \multirow{2}{*}{ Total } \\
\hline & & $\mathbf{V U}$ & DD & $\mathbf{E N}$ & $\mathbf{L C}$ & $\mathbf{N E}$ & NT & \\
\hline \multirow{5}{*}{ Angiosperm } & Climber & 4 & 1 & 2 & 40 & 6 & 10 & 63 \\
\hline & herb & 4 & 1 & 3 & 109 & 22 & 5 & 144 \\
\hline & Shrubs & 4 & 2 & 1 & 66 & 15 & 10 & 98 \\
\hline & Tree & 12 & 3 & 0 & 98 & 20 & 7 & 140 \\
\hline & Epiphytes & 0 & 0 & 1 & 3 & 0 & 1 & 5 \\
\hline \multirow{2}{*}{ Pteridophytes } & Epiphytes & 0 & 0 & 0 & 4 & 1 & 1 & 6 \\
\hline & Fern & 0 & 1 & 0 & 6 & 0 & 0 & 7 \\
\hline \multicolumn{2}{|c|}{ Grand Total } & 24 & 8 & 7 & 326 & 64 & 34 & 463 \\
\hline
\end{tabular}

Table 3. List of Pteridophytes recorded from Karnaphuli range, Kaptai, Bangladesh.

\begin{tabular}{|c|c|c|c|c|c|c|}
\hline$S l$ & Local Name & Botanical Name & Family & Habit & $C S$ & $\begin{array}{l}\text { Specimen } \\
\text { No }\end{array}$ \\
\hline 1. & Guscho Dhekia & Asplenium nidus $\mathrm{L}$. & Asplaniaceae & Epiphytes & $\mathrm{LC}$ & P20173110 \\
\hline 2. & Goyalelata & Adiantum philippense L. & Adiantaceae & Epiphytes & $\mathrm{LC}$ & P20173112 \\
\hline 3. & Hatir khoch & Angiopteris evecta (G.Forst.) Hoffm. & Marattiaceae & Epiphytes & $\mathrm{LC}$ & P20173118 \\
\hline 4. & Pakha Dhekia & Dipteris wallichii (R. Br.) T. Moore & Dipteridaceae & Epiphytes & NT & P20173113 \\
\hline 5. & Pasha Dhekia & $\begin{array}{c}\text { Drymoglossum piloselloides (L.) C. } \\
\text { Presl }\end{array}$ & Dryopteridaceae & Epiphytes & $\mathrm{LC}$ & P20173115 \\
\hline 6. & Pankhiraj & Drynaria quercifolia (L.) J. Sm. & Drynariaceae & Epiphytes & $\mathrm{LC}$ & P20173114 \\
\hline 7. & Dhekishak & Dryopteris filix-mas (L.) Schott & Dryopteridaceae & Fern & $\mathrm{LC}$ & P20173116 \\
\hline 8. & Shada Dhekia & $\begin{array}{c}\text { Helminthostachys zeylanica (L.) } \\
\text { Hook. }\end{array}$ & Ophioglossaceae & Fern & DD & P20173119 \\
\hline 9. & Lata dhekia & Lygodium flexuosum (L.) Sw. & Lygodiaceae & Fern & $\mathrm{LC}$ & P20173117 \\
\hline 10. & Ensiteris & Pteris ensiformis Burm.f. & Pteridaceae & Fern & $\mathrm{LC}$ & P20173122 \\
\hline 11. & Dhekia & Pteris vittata $\mathrm{L}$. & Acrostichaceae & Fern & $\mathrm{LC}$ & P20173111 \\
\hline 12. & Not known & Pyrrosia lanceolata (L.) Farw. & Polypodiaceae & Epiphytes & NE & P20173120 \\
\hline 13. & $\begin{array}{l}\text { Lomba ghashi } \\
\text { fern }\end{array}$ & Vittaria elongate Sw. & Pteridaceae & Epiphytes & $\mathrm{LC}$ & P20173121 \\
\hline
\end{tabular}

CS=Conservation Status; LC=Least concern; NT=Near threatened; VU=Vulnearable; EN=Endangered; $\mathrm{DD}=$ Data Deficient; NE=Not evaluated

Table 4. List of angiosperm flora recorded from Karnaphuli range, Kaptai, Bangladesh.

\begin{tabular}{|c|c|c|c|c|c|c|}
\hline $\begin{array}{c}\text { Sl } \\
\text { No }\end{array}$ & Local Name & Botanical Name & Family & Habit & CS & $\begin{array}{c}\text { Specimen } \\
\text { No }\end{array}$ \\
\hline 1. & Kalomegh & Andrographis paniculata (Brum. f.) & Acanthaceae & Herb & $\mathrm{LC}$ & 20172537 \\
\hline 2. & Kala Jatee Ful & Eranthemum pulchellum Andrews & Acanthaceae & Shrub & $\mathrm{NE}$ & 20172680 \\
\hline 3. & Nilnishinda & Justicia gendarussa Burm. & Acanthaceae & Shrub & $\mathrm{LC}$ & 20172744 \\
\hline 4. & Kalkapana & Rhinacanthus calcaratus Nees & Acanthaceae & Shrub & $\mathrm{NE}$ & 20172862 \\
\hline 5. & Not Known & Staurogyne argentea Wall. & Acanthaceae & Herb & $\mathrm{NE}$ & 20172891 \\
\hline 6. & Ghontolata & Thunbergia alata Bojer ex sims & Acanthaceae & Climber & $\mathrm{NE}$ & 20172923 \\
\hline 7. & Nil lata & Thunbergia grandiflora Roxb & Acanthaceae & Climber & $\mathrm{LC}$ & 20172924 \\
\hline 8. & Dalup & Saurauia roxburghii Wall. & Actinidiaceae & Tree & $\mathrm{LC}$ & 20172871 \\
\hline 9. & Not Known & Dracaena terniflora Roxb. & Agavaceae & Shrub & $\mathrm{LC}$ & 20172673 \\
\hline 10. & Ghritokumari & Aloe vera (L.) Burm.f. & Aloeaceae & Herb & $\mathrm{LC}$ & 20172523 \\
\hline 11. & Apang & Achyranthes aspera L. & Amaranthaceae & Herb & $\mathrm{LC}$ & 20172508 \\
\hline 12. & Helenchan & Alternanthera philoxeroides Kunth. & Amaranthaceae & Herb & $\mathrm{LC}$ & 20172528 \\
\hline 13. & Data shak & Amaranthus dubius Mart. Ex Thellung & Amaranthaceae & Herb & $\mathrm{LC}$ & 20172529 \\
\hline 14. & Lalshak & Amaranthus gangeticus L. & Amaranthaceae & Herb & $\mathrm{LC}$ & 20172530 \\
\hline 15. & Kata notey & Amaranthus spinosus L. & Amaranthaceae & Herb & $\mathrm{LC}$ & 20172531 \\
\hline 16. & Notey & Amaranthus viridis L. & Amaranthaceae & Herb & $\mathrm{LC}$ & 20172532 \\
\hline 17. & $\begin{array}{c}\text { Sada } \\
\text { morogphul }\end{array}$ & Celosia argentea $\mathrm{L}$. & Amaranthaceae & Shrub & $\mathrm{LC}$ & 20172593 \\
\hline 18. & Kaju Badam & Anacardium occidentale L. & Anacardiaceae & Tree & $\mathrm{LC}$ & 20172536 \\
\hline
\end{tabular}




\begin{tabular}{|c|c|c|c|c|c|c|}
\hline $\begin{array}{c}\text { Sl } \\
\text { No }\end{array}$ & Local Name & Botanical Name & Family & Habit & CS & $\begin{array}{l}\text { Specimen } \\
\text { No }\end{array}$ \\
\hline 19. & Boiragula & Holigarna caustica (Dennst.) Oken & Anacardiaceae & Tree & $\mathrm{LC}$ & 20172723 \\
\hline 20. & Jhawa, Barola & $\begin{array}{c}\text { Holigarna longifolia Buch.-Hum. Ex } \\
\text { Roxb. }\end{array}$ & Anacardiaceae & Tree & DD & 20172724 \\
\hline 21. & Am & Mangifera indica $\mathrm{L}$. & Anacardiaceae & Tree & $\mathrm{LC}$ & 20172776 \\
\hline 22. & Uri am & Mangifera sylvatica Roxb. & Anacardiaceae & Tree & VU & 20172777 \\
\hline 23. & Bhela & Semecarpus anacardium L.f. & Anacardiaceae & Tree & $\mathrm{NE}$ & 20172875 \\
\hline 24. & Jangli amra & Spondias indica (L.f.) Kurz & Anacardiaceae & Tree & NT & 20172888 \\
\hline 25. & Atah & Annona reticulata $\mathrm{L}$. & Annonaceae & Shrub & $\mathrm{LC}$ & 20172539 \\
\hline 26. & Sotoyalang & Desmos chinensis Lour. & Annonaceae & Shrub & DD & 20172655 \\
\hline 27. & Not Known & Desmos longiflorus (Roxb.) Saff. & Annonaceae & Tree & $\mathrm{LC}$ & 20172656 \\
\hline 28. & Tasbi & $\begin{array}{c}\text { Miliusa dioeca (Roxb.) Chaowasku \& } \\
\text { Kessler }\end{array}$ & Annonaceae & Tree & $\mathrm{LC}$ & 20172790 \\
\hline 29. & Panjon & $\begin{array}{l}\text { Polyalthia cerasoides (Roxb.) Benth. } \\
\text { \& Hook.f. ex. Bedd. }\end{array}$ & Annonaceae & Tree & $\mathrm{NE}$ & 20172842 \\
\hline 30. & Thankuni & Centella asisatica (L.) Urban & Apiaceae & Herb & $\mathrm{LC}$ & 20172594 \\
\hline 31. & Dhane pata & Coriandrum sativum L. & Apiaceae & Herb & $\mathrm{LC}$ & 20172628 \\
\hline 32. & $\begin{array}{l}\text { Wild } \\
\text { Coriander }\end{array}$ & Eryngium foetidum $\mathrm{L}$. & Apiaceae & Herb & $\mathrm{NE}$ & 20172681 \\
\hline 33. & Crape jasmine & Abernaemonlana divaricata (L.) Br. & Apocynaceae & Shrub & $\mathrm{LC}$ & 20172503 \\
\hline 34. & Pani Dul & Anodendron paniculatum A. DC. & Apocynaceae & Climber & $\mathrm{NE}$ & 20172540 \\
\hline 35. & Nayantara & Catharanthus roseus (L.) G. Don & Apocynaceae & Herb & $\mathrm{LC}$ & 20172589 \\
\hline 36. & Kurch/kuruj & $\begin{array}{l}\text { Holarrhena pubescence (Buch. - } \\
\text { Hum.) Wall ex. G. Don }\end{array}$ & Apocynaceae & Tree & $\mathrm{LC}$ & 20172722 \\
\hline 37. & Chita & Marsdenia tenacissima (Roxb.) Moon & Apocynaceae & Shrub & VU & 20172780 \\
\hline 38. & Sarpagandha & $\begin{array}{c}\text { Rauvolfia serpentina (L.) Benth. ex } \\
\text { Kurz }\end{array}$ & Apocynaceae & Shrub & NT & 20172859 \\
\hline 39. & Shukh tagar & $\begin{array}{c}\text { Tabernaemontana corymbosa Roxb. } \\
\text { ex Wall. }\end{array}$ & Apocynaceae & Shrub & $\mathrm{LC}$ & 20172909 \\
\hline 40. & jangli tagar & $\begin{array}{l}\text { Tabernaemontana divaricata (L.) } \\
\text { R.Br. ex Roem. \& Schult. }\end{array}$ & Apocynaceae & Shrub & $\mathrm{LC}$ & 20172910 \\
\hline 41. & Lata am & Willughbeia edulis Roxb. & Apocynaceae & Climber & VU & 20172940 \\
\hline 42. & Indro job & Wrightia arborea (Dennst.) Mabb. & Apocynaceae & Tree & NT & 20172942 \\
\hline 43. & Satagoza & Ilex umbellulata (Wall.) Loes. & aquifoliaceae & Tree & $\mathrm{NE}$ & 20172732 \\
\hline 44. & Nimahook & Aglaonema hookerianum Schott. & Araceae & Herb & DD & 20172514 \\
\hline 45. & Man kochu & Alocacia indica (Roxb.) Schott. & Araceae & Shrub & $\mathrm{LC}$ & 20172519 \\
\hline 46. & Chinese Taro & Alocasia cucullata (Lour.) Schott & Araceae & Herb & $\mathrm{LC}$ & 20172520 \\
\hline 47. & Voodoo lily & Alocasia fornicata (Roxb.) Schott. & Araceae & Herb & $\mathrm{LC}$ & 20172521 \\
\hline 48. & Mankachu & Alocasia macrorrhizos (L.) G. Don & Araceae & Herb & LC & 20172522 \\
\hline 49. & Ol kachu & $\begin{array}{l}\text { Amorphophallus campanulatus } \\
\text { (Roxb.) Bl. }\end{array}$ & Araceae & Herb & $\mathrm{LC}$ & 20172534 \\
\hline 50 & Kochu & Colocasia esculanta (L.) Schott. & Araceae & Herb & $\mathrm{LC}$ & 20172622 \\
\hline 51. & Homalomena & $\begin{array}{c}\text { Homalomena aromatica (Spreng.) } \\
\text { Schott. }\end{array}$ & Araceae & Herb & VU & 20172725 \\
\hline 52. & Kata kachu & Lasia spinosa (L.) Thwaites & Araceae & Herb & $\mathrm{LC}$ & 20172755 \\
\hline 53. & Hatilata & Pothos scandens L. & Araceae & Climber & $\mathrm{LC}$ & 20172844 \\
\hline 54. & Fidoca & $\begin{array}{c}\text { Rhaphidophora glauca (Wallich) } \\
\text { Schott }\end{array}$ & Araceae & Climber & VU & 20172860 \\
\hline 55. & Hongkongifido & Rhaphidophora hongkongensis Schott & Araceae & Herb & $\mathrm{VU}$ & 20172861 \\
\hline 56. & Ghetkochu & $\begin{array}{c}\text { Typhonium trilobatum (L.) Schott var. } \\
\text { trilobatun }\end{array}$ & Araceae & Herb & $\mathrm{LC}$ & 20172931 \\
\hline 57. & Pani kesuri & Macropanax dispermus (B1.) Kuntze & Araliaceae & Tree & NT & 20172772 \\
\hline 58. & Jeng jil & Schefflera elliptica (Blume) Harms. & Araliaceae & Tree & $\mathrm{LC}$ & 20172873 \\
\hline 59. & Gracifuli & Didymosperma gracilis Hook. F. & Arecaceae & Herb & NT & 20172658 \\
\hline 60. & Isharmul & Aristolochia tagala Cham. & Aristolochiaceae & Climber & $\mathrm{LC}$ & 20172551 \\
\hline 61. & Baro akanda & Calotropis gigantea (L.) Ait.f. & Ascelpiadaceae & Shrub & $\mathrm{LC}$ & 20172578 \\
\hline 62. & Anantamul & Hemidesmus indicus (L.) R. Br. & Ascelpiadaceae & Climber & $\mathrm{LC}$ & 20172718 \\
\hline 63. & Not known & $\begin{array}{c}\text { Myriopteron extensum (Wright \& } \\
\text { Arn.) K. Schums. }\end{array}$ & Ascelpiadaceae & Climber & $\mathrm{EN}$ & 20172802 \\
\hline 64. & Numrula & Dischidia nummularia R. Br. & Asclepiadaceae & Epiphytes & EN & 20172671 \\
\hline
\end{tabular}




\begin{tabular}{|c|c|c|c|c|c|c|}
\hline $\begin{array}{c}\text { Sl } \\
\text { No }\end{array}$ & Local Name & Botanical Name & Family & Habit & CS & $\begin{array}{c}\text { Specimen } \\
\text { No } \\
\end{array}$ \\
\hline 65. & Serapatahoya & $\begin{array}{c}\text { Hoya parasitica (Roxb.) Wall. Ex } \\
\text { Wight. }\end{array}$ & Asclepiadaceae & Epiphytes & $\mathrm{LC}$ & 20172728 \\
\hline 66. & Not Known & Dracaena reflexa var. reflexa & Asparagaceae & Shrub & $\mathrm{NE}$ & 20172672 \\
\hline 67. & Nagor chandal & Blumea balsamifera DC. & Asteraceae & Shrub & $\mathrm{LC}$ & 20172567 \\
\hline 68. & Kukur sunga & Blumea lacera (Burn. f.) DC. & Asteraceae & Herb & $\mathrm{LC}$ & 20172568 \\
\hline 69. & Assam lata & Chromolaena odorata L. & Asteraceae & Shrub & $\mathrm{LC}$ & 20172597 \\
\hline 70. & $\begin{array}{l}\text { Ayapan, shial } \\
\text { muthi }\end{array}$ & Eupatorium ayapana Vahl. & Asteraceae & Herb & $\mathrm{LC}$ & 20172683 \\
\hline 71. & Nurapali & Gynura nepalensis A. DC. & Asteraceae & Herb & $\mathrm{LC}$ & 20172711 \\
\hline 72. & Not known & Mikania cordata (Burm. f.) B.L.Rob. & Asteraceae & Climber & $\mathrm{LC}$ & 20172788 \\
\hline 73. & Asam lata & Mikania micrantha Kunth & Asteraceae & Shrub & $\mathrm{LC}$ & 20172789 \\
\hline 74. & Marhatitiga & Spilanthes acmella L. & Asteraceae & Herb & $\mathrm{LC}$ & 20172887 \\
\hline 75. & Kukshim & Vernonia patula (Aiton) Merr & Asteraceae & Herb & $\mathrm{LC}$ & 20172936 \\
\hline 76. & Bhimra & Wedelia chinensis (Osbeck) Merr & Asteraceae & Herb & $\mathrm{LC}$ & 20172939 \\
\hline 77. & Ghagra & Xanthium indicum J. Konig. Ex Roxb. & Asteraceae & Herb & $\mathrm{LC}$ & 20172943 \\
\hline 78. & Pui shak & Basella rubra $\mathrm{L}$. & Basellaceae & Climber & $\mathrm{LC}$ & 20172559 \\
\hline 79. & Not known & Begonia picta $\mathrm{Sm}$. & Begoniaceae & Herb & $\mathrm{NE}$ & 20172562 \\
\hline 80 . & Gonirakto & Begonia roxburghii (Miq.) A. DC. & Begoniaceae & Herb & $\mathrm{NE}$ & 20172563 \\
\hline 81. & Not known & Begonia thomsonii A.DC. & Begoniaceae & Herb & $\mathrm{NE}$ & 20172564 \\
\hline 82. & Khona & Oroxylum indicum (L.) Kurz & Bignoniaceae & Tree & $\mathrm{LC}$ & 20172813 \\
\hline 83. & Hona & $\begin{array}{l}\text { Pagenalia longifolia (Wild.) K. } \\
\text { Schum. }\end{array}$ & Bignoniaceae & Tree & NT & 20172820 \\
\hline 84. & Dharmara & $\begin{array}{l}\text { Stereospermum personatum (Hassk.) } \\
\text { Chatt. }\end{array}$ & Bignoniaceae & Tree & $\mathrm{LC}$ & 20172897 \\
\hline 85. & Parul & $\begin{array}{c}\text { Stereospermum suaveolens (Roxb.) } \\
\text { DC. }\end{array}$ & Bignoniaceae & Tree & $\mathrm{LC}$ & 20172898 \\
\hline 86. & Ban shimul & Bombax insigne Wall. & Bombacaceae & Tree & $\mathrm{LC}$ & 20172571 \\
\hline 87. & Bohul & Cordia dichotoma $\mathrm{G}$. Forst. & Boraginaceae & Tree & $\mathrm{LC}$ & 20172627 \\
\hline 88. & Hatishor & Heliotropium indicum $\mathrm{L}$. & Boraginaceae & Herb & $\mathrm{LC}$ & 20172717 \\
\hline 89. & Dhup & Canarium resiniferum Bruce ex King & Burseraceae & Tree & DD & 20172579 \\
\hline 90. & Jeol bhadi & Garuga pinnata Roxb. & Burseraceae & Tree & $\mathrm{LC}$ & 20172701 \\
\hline 91. & gutgutia, neul & $\begin{array}{c}\text { Protium serratum (Wall. ex Coelbr.) } \\
\text { Engl }\end{array}$ & Burseraceae & Tree & $\mathrm{LC}$ & 20172850 \\
\hline 92. & Sada kanchan & Bauhinia acuminata $\mathrm{L}$. & Caesalpiniaceae & Shrub & $\mathrm{LC}$ & 20172560 \\
\hline 93. & $\begin{array}{l}\text { Rakta } \\
\text { Kanchan }\end{array}$ & Bauhinia variegata $\mathrm{L}$. & Caesalpiniaceae & Tree & $\mathrm{LC}$ & 20172561 \\
\hline 94. & $\begin{array}{l}\text { Bogaserra } \\
\text { kanta }\end{array}$ & Caesalpinia cucullata Roxb. & Caesalpiniaceae & Tree & $\mathrm{LC}$ & 20172575 \\
\hline 95. & Bador lati & Cassia fistula L. & Caesalpiniaceae & Tree & $\mathrm{LC}$ & 20172585 \\
\hline 96. & Kalkasunde & Cassia occidentalis $\mathrm{L}$. & Caesalpiniaceae & Shrub & $\mathrm{LC}$ & 20172586 \\
\hline 97. & Ashok & Saraca asoca (Roxb.) Willd. & Caesalpiniaceae & Tree & $\mathrm{LC}$ & 20172869 \\
\hline 98. & Dadmardan & Senna alata (L.) Roxb. & Caesalpiniaceae & Shrub & $\mathrm{LC}$ & 20172876 \\
\hline 99. & Gandhosena & Senna hirsuta L. & Caesalpiniaceae & Herb & $\mathrm{LC}$ & 20172877 \\
\hline 100. & Dadmari & Senna tora (L.) Roxb. & Caesalpiniaceae & Herb & $\mathrm{LC}$ & 20172879 \\
\hline 101. & Tetul & Tamarindus indica $\mathrm{L}$. & Caesalpiniaceae & Tree & $\mathrm{LC}$ & 20172914 \\
\hline 102. & Bhang & Cannabis sativus L. & Cannabaceae & Herb & $\mathrm{LC}$ & 20172580 \\
\hline 103. & Ladum, Barun & Crataeva magna (Lour.) DC. & Capparaceae & Tree & $\mathrm{NE}$ & 20172630 \\
\hline 104. & Madhu matoli & $\begin{array}{c}\text { Stixis suaveolens (Roxburgh) Pierre, } \\
\text { Bull. }\end{array}$ & Capparaceae & Climber & VU & 20172899 \\
\hline 105. & Haldehurhurey & Cleome viscosa $\mathrm{L}$. & Capparidaceae & Herb & $\mathrm{LC}$ & 20172613 \\
\hline 106. & Papaya & Carica papaya $\mathrm{L}$. & Caricaceae & Shrub & $\mathrm{LC}$ & 20172583 \\
\hline 107. & Kurima & Bhesa robusta (Roxb.) Ding Hou & Celastraceae & Tree & VU & 20172565 \\
\hline 108. & Bathua shak & Chenopodium album L. & Chenopodiaceae & Herb & LC & 20172595 \\
\hline 109. & Esri & $\begin{array}{c}\text { Anogeissus acuminata (Roxb. ex DC.) } \\
\text { Wall. ex Guill. \& Perr. }\end{array}$ & Combretaceae & Tree & LC & 20172541 \\
\hline 110. & Arjun & $\begin{array}{c}\text { Terminalia arjuna (Roxb. ex DC.) } \\
\text { Wight \& Arn }\end{array}$ & Combretaceae & Tree & VU & 20172917 \\
\hline 111. & Bohera & Terminalia belerica (Gaertn.) Roxb. & Combretaceae & Tree & $\mathrm{LC}$ & 20172918 \\
\hline 112. & Katbadam & Terminalia catappa $\mathrm{L}$ & Combretaceae & Tree & $\mathrm{LC}$ & 20172919 \\
\hline
\end{tabular}




\begin{tabular}{|c|c|c|c|c|c|c|}
\hline $\begin{array}{c}\text { Sl } \\
\text { No }\end{array}$ & Local Name & Botanical Name & Family & Habit & CS & $\begin{array}{c}\text { Specimen } \\
\text { No } \\
\end{array}$ \\
\hline 113. & Horitoki & Terminalia chebula (Gaertn.) Retz. & Combretaceae & Tree & VU & 20172920 \\
\hline 114. & na & $\begin{array}{c}\text { Amischotolype hookeri Hassk.) H. } \\
\text { Hara }\end{array}$ & Commelinaceae & Herb & $\mathrm{NE}$ & 20172533 \\
\hline 115. & Dholpata & Commelina benghalensis L. & Commelinaceae & Herb & $\mathrm{LC}$ & 20172623 \\
\hline 116. & $\begin{array}{c}\text { Monayna } \\
\text { kanshira }\end{array}$ & Commelina diffusa Burm. $\mathrm{f}$. & Commelinaceae & Herb & $\mathrm{LC}$ & 20172624 \\
\hline 117. & Not Known & Floscopa scandens Lour. & Commelinaceae & Herb & $\mathrm{LC}$ & 20172698 \\
\hline 118. & Not Known & Connarus wightii Hook. f. & Connaraceae & Shrub & $\mathrm{NE}$ & 20172625 \\
\hline 119. & Pashacuta & Rourea commutata Planch. & Connaraceae & Shrub & $\mathrm{LC}$ & 20172866 \\
\hline 120. & Napigach & Peliosanthes teta Andrews & Convallariaceae & Herb & $\mathrm{LC}$ & 20172825 \\
\hline 121. & $\begin{array}{l}\text { Silky Morning } \\
\text { Glory }\end{array}$ & Argyreia sericea Dalz. \& Gib. & Convolvulaceae & Climber & $\mathrm{NE}$ & 20172550 \\
\hline 122. & Bhui okra & Evolvulus nummularius L. & Convolvulaceae & Herb & $\mathrm{LC}$ & 20172685 \\
\hline 123. & $\begin{array}{c}\text { Kalmi } \\
\operatorname{shak}(\mathrm{B})\end{array}$ & Ipomoea aquatica Forssk. & Convolvulaceae & Herb & $\mathrm{LC}$ & 20172733 \\
\hline 124. & Dhol kalmi & Ipomoea fistulosa Mart. ex Chorsy & Convolvulaceae & Shrub & $\mathrm{LC}$ & 20172734 \\
\hline 125. & $\begin{array}{l}\text { Languli lata } \\
\text { kalmi }\end{array}$ & Ipomoea pestigridis $\mathrm{L}$. & Convolvulaceae & Herb & $\mathrm{LC}$ & 20172735 \\
\hline 126. & Sada kolmi & Merremia umbellata (L.) Hallier f. & Convolvulaceae & Climber & $\mathrm{LC}$ & 20172784 \\
\hline 127. & Kusta & Costus speciosus (J.Koenig) Sm. & Costaceae & Shrub & $\mathrm{LC}$ & 20172629 \\
\hline 128. & Mula shak & Raphanus sativus L. & Cruciferae & Herb & $\mathrm{LC}$ & 20172858 \\
\hline 129. & telacucha & Coccinia grandis (L.) Voigt & Cucurbitaceae & Climber & $\mathrm{LC}$ & 20172619 \\
\hline 130. & katabangi & Cucumis hystrix Chakrav. & Cucurbitaceae & Climber & $\mathrm{EN}$ & 20172637 \\
\hline 131. & Tikkur Halud & Curcuma angustifolia Roxb. & Cucurbitaceae & Climber & $\mathrm{LC}$ & 20172639 \\
\hline 132. & Not Known & $\begin{array}{l}\text { Gymnopetalum cochinchinense } \\
\text { (Lour.) kutz }\end{array}$ & Cucurbitaceae & Climber & $\mathrm{LC}$ & 20172710 \\
\hline 133. & Lau shak & Lagenaria siceraria (Molina) Standl. & Cucurbitaceae & Climber & $\mathrm{LC}$ & 20172751 \\
\hline 134. & Kakro & Momodica dioca Roxb. ex Willd. & Cucurbitaceae & Climber & $\mathrm{LC}$ & 20172793 \\
\hline 135. & Karala & Momordica charantia L. & Cucurbitaceae & Climber & $\mathrm{LC}$ & 20172794 \\
\hline 136. & Ban kakrol & Momordica cochinchinensis Lour. & Cucurbitaceae & Climber & $\mathrm{LC}$ & 20172795 \\
\hline 137. & Not known & Momordica foetida Schumach. & Cucurbitaceae & Climber & $\mathrm{NE}$ & 20172796 \\
\hline 138. & Rakhal Gota & Solena amplexicaulis (Lam.) Gandhi & Cucurbitaceae & Herb & $\mathrm{LC}$ & 20172886 \\
\hline 139. & Makal & Trichosanthes tricuspidata Lour. & Cucurbitaceae & Climber & $\mathrm{LC}$ & 20172930 \\
\hline 140. & Kassari & $\begin{array}{c}\text { Actinoscirpus grossus (L.f) Goetgh. \& } \\
\text { D.A. Simpson }\end{array}$ & Cyperaceae & Herb & $\mathrm{LC}$ & 20172511 \\
\hline 141. & Alga ghasi & Cyperus laxus Lam. & Cyperaceae & Herb & $\mathrm{LC}$ & 20172646 \\
\hline 142. & Sakto khagra & Cyperus pilosus Vahi. var. Pilosus & Cyperaceae & Herb & $\mathrm{LC}$ & 20172647 \\
\hline 143. & Phyla fimbry & $\begin{array}{c}\text { Fimbristylis aphylla Steud. } \\
\text { Kyllinga nemoralis (J. R. Forster \& G. }\end{array}$ & Cyperaceae & Herb & LC & 20172693 \\
\hline 144. & Nirvishi & $\begin{array}{c}\text { Forster) Dandy ex Hutchinson \& } \\
\text { Dalziel }\end{array}$ & Cyperaceae & Herb & $\mathrm{LC}$ & 20172750 \\
\hline 145. & Dharal ghasi & Scleria terrestris (L.) Fassett. & Cyperaceae & Herb & $\mathrm{LC}$ & 20172874 \\
\hline 146. & moacura & $\begin{array}{c}\text { Dichapetalum gelonioides (Roxb.) } \\
\text { Engl }\end{array}$ & Dichapetalaceae & Tree & $\mathrm{NE}$ & 20172657 \\
\hline 147. & Chalta & Dillenia indica $\mathrm{L}$. & Dilleniaceae & Tree & $\mathrm{LC}$ & 20172659 \\
\hline 148. & Hargaza, ajuli & Dillenia pentagyna Roxb. & Dilleniaceae & Tree & $\mathrm{LC}$ & 20172660 \\
\hline 149. & Lata chalta & Tetracera sarmentosa (L.) Vahl. & Dilleniaceae & Shrub & $\mathrm{LC}$ & 20172921 \\
\hline 150. & Gasalu & Dioscorea alata $\mathrm{L}$. & Dioscoreaceae & Climber & $\mathrm{LC}$ & 20172661 \\
\hline 151. & Not known & Dioscorea bulbifera $\mathrm{L}$. & Dioscoreaceae & Climber & $\mathrm{LC}$ & 20172662 \\
\hline 152. & Shora alu & Dioscorea glabra Roxb. & Dioscoreaceae & Climber & VU & 20172663 \\
\hline 153. & $\begin{array}{l}\text { Milanomaittya } \\
\text { alu }\end{array}$ & $\begin{array}{c}\text { Dioscorea melanophyma Prain \& } \\
\text { Burkill }\end{array}$ & Dioscoreaceae & Climber & NT & 20172664 \\
\hline 154. & Not known & Dioscorea pentaphylla L. & Dioscoreaceae & Climber & $\mathrm{LC}$ & 20172665 \\
\hline 155. & Dholi garjan & Dipterocarpus alatus Roxb. & Dipterocarpaceae & Tree & $\mathrm{LC}$ & 20172669 \\
\hline 156. & teli garjan & Dipterocarpus turbinatus Gaertn. & Dipterocarpaceae & Tree & $\mathrm{LC}$ & 20172670 \\
\hline 157. & Telsur & Hopea odorata Roxb. & Dipterocarpaceae & Tree & VU & 20172726 \\
\hline 158. & Bilati gub & Diospyros blancoi A. DC. & Ebenaceae & Tree & $\mathrm{LC}$ & 20172666 \\
\hline 159. & Gub & Diospyros malabarica (Desr.) Kostel. & Ebenaceae & Tree & $\mathrm{LC}$ & 20172667 \\
\hline 160. & Uri Gub & Diospyros ramiflora Roxb. & Ebenaceae & Tree & VU & 20172668 \\
\hline
\end{tabular}




\begin{tabular}{|c|c|c|c|c|c|c|}
\hline $\begin{array}{r}\text { Sl } \\
\text { No }\end{array}$ & Local Name & Botanical Name & Family & Habit & $\mathbf{C S}$ & $\begin{array}{l}\text { Specimen } \\
\text { No }\end{array}$ \\
\hline 161. & Jolpai & Elaeocarpus floribundus Blume & Elaeocarpaceae & Tree & $\mathrm{LC}$ & 20172677 \\
\hline 162. & Muktajhuri & Acalypha indica $\mathrm{L}$. & Euphorbiaceae & Herb & $\mathrm{LC}$ & 20172506 \\
\hline 163. & Lalsa & Actephila excelsa (Dalzell) Mull.Arg. & Euphorbiaceae & Tree & $\mathrm{LC}$ & 20172510 \\
\hline 164. & Choto kechua & $\begin{array}{c}\text { Aporosa octandra (Buch.-Ham. Ex D. } \\
\text { Don) A. R. Vickery }\end{array}$ & Euphorbiaceae & Tree & $\mathrm{NE}$ & 20172545 \\
\hline 165. & Silpati & Breynia retusa (Dennst.) Alston & Euphorbiaceae & Shrub & $\mathrm{NE}$ & 20172572 \\
\hline 166. & Kantokushi & Bridelia retusa L. & Euphorbiaceae & Tree & $\mathrm{NE}$ & 20172573 \\
\hline 167. & Paharibichuti & Cnesmone javanica Bl. & Euphorbiaceae & Shrub & $\mathrm{NE}$ & 20172618 \\
\hline 168. & Bankhira & Croton bonplandianum Baill. & Euphorbiaceae & Herb & $\mathrm{LC}$ & 20172636 \\
\hline 169. & Dudhiya & Euphorbia hirta L. & Euphorbiaceae & Herb & $\mathrm{LC}$ & 20172684 \\
\hline 170. & Not Known & $\begin{array}{c}\text { Glochidion zeylanicum (Gaertn.) A. } \\
\text { Juss. }\end{array}$ & Euphorbiaceae & Tree & VU & 20172705 \\
\hline 171. & Ban bherenda & Jatropha curcas L. & Euphorbiaceae & Shrub & $\mathrm{LC}$ & 20172742 \\
\hline 172. & Lal bherenda & Jatropha gossypifolia L. & Euphorbiaceae & Shrub & $\mathrm{LC}$ & 20172743 \\
\hline 173. & Bura & $\begin{array}{c}\text { Macaranga denticulata (Blume.) } \\
\text { Muell. Arg. }\end{array}$ & Euphorbiaceae & Tree & $\mathrm{LC}$ & 20172771 \\
\hline 174. & Kumaribura & $\begin{array}{c}\text { Mallotus tetracoccus (Roxburgh) } \\
\text { Kurz }\end{array}$ & Euphorbiaceae & Tree & $\mathrm{LC}$ & 20172775 \\
\hline 175. & Not known & Pedilanthus tithymaloides (L.) Poiteau & Euphorbiaceae & Shrub & $\mathrm{LC}$ & 20172824 \\
\hline 176. & Verenda & Ricinus communis L. & Euphorbiaceae & Herb & $\mathrm{LC}$ & 20172865 \\
\hline 177. & Moricha & Suregada multiflora (A. Juss.) Baill. & Euphorbiaceae & Tree & $\mathrm{LC}$ & 20172901 \\
\hline 178. & Pitali & Trewia nudiflora L. & Euphorbiaceae & Tree & $\mathrm{LC}$ & 20172929 \\
\hline 179. & Tetuiya Koroi & Albizia odoratissima (L.f.) Benth. & Fabaceae & Tree & $\mathrm{LC}$ & 20172516 \\
\hline 180. & Sil Koroi & Albizia procera (Roxb.) Benth. & Fabaceae & Tree & $\mathrm{LC}$ & 20172517 \\
\hline 181. & Arhar & Cajanus cajan (L.) Huth & Fabaceae & Shrub & $\mathrm{LC}$ & 20172576 \\
\hline 182. & $\begin{array}{l}\text { Chhoto } \\
\text { Jhunjhuni }\end{array}$ & Crotalaria incana L. & Fabaceae & Herb & $\mathrm{NE}$ & 20172633 \\
\hline 183. & Shonpot & Crotalaria juncea L. & Fabaceae & Tree & $\mathrm{LC}$ & 20172634 \\
\hline 184. & Jhunjhuni & Crotalaria pallida Aiton. & Fabaceae & Herb & $\mathrm{NE}$ & 20172635 \\
\hline 185. & Chalani & Desmodium gangeticum (L.) DC. & Fabaceae & Herb & $\mathrm{LC}$ & 20172651 \\
\hline 186. & Desmodium & Desmodium heterocarpon (L.) DC. & Fabaceae & Herb & NT & 20172652 \\
\hline 187. & laxmodi & Desmodium laxiflorum $D C$. & Fabaceae & Climber & $\mathrm{LC}$ & 20172653 \\
\hline 188. & Kalaliya & Desmodium trifolium (L.) DC. & Fabaceae & Herb & $\mathrm{NE}$ & 20172654 \\
\hline 189. & Mandar & Erythrina variegata L. var. Variegata & Fabaceae & Tree & $\mathrm{LC}$ & 20172682 \\
\hline 190. & Bara salphan & Flemingia macrophylla (Willd.) Merr. & Fabaceae & Shrub & $\mathrm{LC}$ & 20172695 \\
\hline 191. & Simbusak & Flemingia strobilifera (L.) W.T. Aiton & Fabaceae & Shrub & $\mathrm{LC}$ & 20172696 \\
\hline 192. & Not known & Gliricidia sepium (Jacq.) Walp. & Fabaceae & Tree & $\mathrm{LC}$ & 20172703 \\
\hline 193. & $\begin{array}{l}\text { Ghorachokha } \\
\text { shim }\end{array}$ & Ormosia robusta Baker & Fabaceae & Tree & DD & 20172812 \\
\hline 194. & $\begin{array}{l}\text { Kamranga } \\
\text { seem }\end{array}$ & $\begin{array}{c}\text { Psophocarpus tetragonolobus (L.) } \\
\text { DC. }\end{array}$ & Fabaceae & Climber & $\mathrm{LC}$ & 20172853 \\
\hline 195. & Gola kunch & Pueraria tuberosa (Willd.) DC. & Fabaceae & Climber & NT & 20172856 \\
\hline 196. & $\begin{array}{l}\text { Bara } \\
\text { chalkesunda }\end{array}$ & Senna occidentalis (L.) Link. & Fabaceae & Shrub & $\mathrm{LC}$ & 20172878 \\
\hline 197. & Luri manda & $\begin{array}{l}\text { Tadehagi triquetrum (L.) H. Ohashi } \\
\text { subsp. triquetrum }\end{array}$ & Fabaceae & Shrub & $\mathrm{NE}$ & 20172913 \\
\hline 198. & Ban Nil & Tephrosia purpurea (L.) Pers & Fabaceae & Herb & $\mathrm{LC}$ & 20172916 \\
\hline 199. & Asian foxtail & Uraria crinita (L.) DC. & Fabaceae & Shrub & $\mathrm{NE}$ & 20172933 \\
\hline 200. & Sankarjata & Uraria picta Desv. & Fabaceae & Shrub & $\mathrm{LC}$ & 20172934 \\
\hline 201. & Batna & Castanopsis armata (Roxb.) Spach & Fagaceae & Tree & $\mathrm{NE}$ & 20172587 \\
\hline 202. & $\begin{array}{l}\text { Kantasingarh, } \\
\text { kera }\end{array}$ & Castanopsis tribuloides (Sm.) A.DC. & Fagaceae & Tree & $\mathrm{NE}$ & 20172588 \\
\hline 203. & Chilla & Casearia tomentosa Roxb. & Flacortiaceae & Tree & $\mathrm{LC}$ & 20172584 \\
\hline 204. & Chalmugra & Hydnocarpus kurzii (King) Warb. & Flacortiaceae & Tree & NT & 20172729 \\
\hline 205. & Painnya gola & Flacourtia jangomas Lour. & Flacourtiaceae & Tree & $\mathrm{LC}$ & 20172694 \\
\hline 206. & Not Known & $\begin{array}{c}\text { Rhyncotechum ellipticum (Wall ex D. } \\
\text { Dietr.) A. DC. }\end{array}$ & Gesneriaceae & Shrub & NT & 20172864 \\
\hline $\begin{array}{l}207 . \\
208 .\end{array}$ & $\begin{array}{l}\text { Not Known } \\
\text { Not Known }\end{array}$ & $\begin{array}{l}\text { Stauranthera grandiflora Benth. } \\
\text { Stauranthera umbrosa (Griff.) }\end{array}$ & $\begin{array}{l}\text { Gesneriaceae } \\
\text { Gesneriaceae }\end{array}$ & $\begin{array}{l}\text { Herb } \\
\text { Herb }\end{array}$ & $\begin{array}{l}\text { NT } \\
\text { NE }\end{array}$ & $\begin{array}{l}20172889 \\
20172890\end{array}$ \\
\hline
\end{tabular}




\begin{tabular}{|c|c|c|c|c|c|c|}
\hline $\begin{array}{r}\text { Sl } \\
\text { No }\end{array}$ & Local Name & Botanical Name & Family & Habit & CS & $\begin{array}{c}\text { Specimen } \\
\text { No }\end{array}$ \\
\hline & & C.B.Clarke & & & & \\
\hline 209. & Cawagola & Garcinia cowa Roxb. ex DC. & Guttifarae & Tree & $\mathrm{LC}$ & 20172699 \\
\hline 210. & Rigra & Ottelia alismoides (L.) Pers. & Hydrocharitaceae & Herb & $\mathrm{LC}$ & 20172816 \\
\hline 211. & Pudina pata & Mentha viridis $\mathrm{L}$. & Labiatae & Herb & $\mathrm{LC}$ & 20172783 \\
\hline 212. & Ajuga & Ajuga macrosperma Wall. ex Benth. & Lamiaceae & Herb & $\mathrm{NE}$ & 20172515 \\
\hline 213. & Jangli tulsi & Anisomeles indica (L.) Kuntze & Lamiaceae & Herb & $\mathrm{LC}$ & 20172538 \\
\hline 214. & Fire bush & Clerodendrum nutans Wall. ex Jack & Lamiaceae & Shrub & $\mathrm{NE}$ & 20172617 \\
\hline 215. & Red basket & Coleus rehneltianus A. Berger & Lamiaceae & Herb & $\mathrm{LC}$ & 20172620 \\
\hline 216. & Pathor chur & Coleus scutellariodes (L.) Benth & Lamiaceae & Shrub & $\mathrm{LC}$ & 20172621 \\
\hline 217. & Not Known & Dysophylla auricularia B1. & Lamiaceae & Herb & $\mathrm{NE}$ & 20172675 \\
\hline 218. & Jateri bormala & $\begin{array}{c}\text { Gomphostemma parviflorum Wallich } \\
\text { ex Bentham }\end{array}$ & Lamiaceae & Shrub & $\mathrm{LC}$ & 20172708 \\
\hline 219. & Gol tokma & Hyptis brevipes Poit. & Lamiaceae & Herb & $\mathrm{LC}$ & 20172730 \\
\hline 220. & Tokma & Hyptis suaveolens (L.) Poit. & Lamiaceae & Shrub & $\mathrm{LC}$ & 20172731 \\
\hline 221. & Shetodron & Leucas aspera (Roth.) Spreng & Lamiaceae & Herb & $\mathrm{LC}$ & 20172761 \\
\hline 222. & Dando kalosh & Leucas cephalotes (Roth.) Spreng & Lamiaceae & Herb & $\mathrm{LC}$ & 20172762 \\
\hline 223. & Dando kalosh & Leucas indica (L.) R. Br. ex Vatke & Lamiaceae & Herb & $\mathrm{LC}$ & 20172763 \\
\hline 224. & Bantulsi & Ocimum americum $\mathrm{L}$. & Lamiaceae & Herb & $\mathrm{LC}$ & 20172806 \\
\hline 225. & Ram tulsi & Ocimum gratissimum $\mathrm{L}$. & Lamiaceae & Shrub & $\mathrm{LC}$ & 20172807 \\
\hline 226. & Tulsi & Ocimum tenuiflorum $\mathrm{L}$. & Lamiaceae & Shrub & $\mathrm{LC}$ & 20172808 \\
\hline 227. & Bankiker & $\begin{array}{l}\text { Orthosiphon rubicundus (D. Don) } \\
\text { Benth }\end{array}$ & Lamiaceae & Herb & $\mathrm{NE}$ & 20172814 \\
\hline 228. & Paraylla & Perilla frutescens (L.) Britt & Lamiaceae & Herb & $\mathrm{LC}$ & 20172830 \\
\hline 229. & Tejbohol & Cinnamomum cecidodaphne Meisn. & Lauraceae & Tree & NT & 20172599 \\
\hline 230. & Ban Tejpata & Cinnamomum pachyphyllum Kosterm. & Lauraceae & Tree & $\mathrm{NE}$ & 20172600 \\
\hline 231. & Tejpata & $\begin{array}{c}\text { Cinnamomum tamala (Buch.-hum.) } \\
\text { Nees \& Eberm. }\end{array}$ & Lauraceae & Tree & $\mathrm{NE}$ & 20172601 \\
\hline 232. & Baghraj & Dehaasia kurzii King ex Hook. F. & Lauraceae & Tree & $\mathrm{NE}$ & 20172650 \\
\hline 233. & Menda, Meda & Litsea glutinosa Lour. & Lauraceae & Tree & $\mathrm{LC}$ & 20172768 \\
\hline 234. & $\begin{array}{c}\text { Bara } \\
\text { kukurchita }\end{array}$ & Litsea monopetala (Roxb.) Persoon & Lauraceae & Shrub & $\mathrm{LC}$ & 20172769 \\
\hline 235. & Parabatpadi & Leea aequata $\mathrm{L}$. & Leeaceae & Shrub & $\mathrm{LC}$ & 20172757 \\
\hline 236. & Danakhonghla & Leea alata Edgen. & Leeaceae & Shrub & $\mathrm{LC}$ & 20172758 \\
\hline 237. & Dholshomdro & Leea macrophylla Roxb. ex Hornem & Leeaceae & Shrub & $\mathrm{LC}$ & 20172759 \\
\hline 238. & Lalbhanga & Leea rubra Blume ex Spreng. & Leeaceae & Shrub & ne & 20172760 \\
\hline 239. & Barakanur & Crinum asiaticum $\mathrm{L}$. & Liliaceae & Herb & $\mathrm{LC}$ & 20172632 \\
\hline 240. & Talamuli & Curculigo orchioides Gaertn. & Liliaceae & Herb & $\mathrm{LC}$ & 20172638 \\
\hline 241. & Satipata & Molineria capitulata (Lour.)Herb. & Liliaceae & Herb & $\mathrm{LC}$ & 20172792 \\
\hline 242. & Zai ghas & Lindernia antipoda (1.) Alston & Linderniaceae & Herb & $\mathrm{LC}$ & 20172765 \\
\hline 243. & Not Known & Lindernia ciliata (Colsm.) Pennell & Linderniaceae & Herb & $\mathrm{LC}$ & 20172766 \\
\hline 244. & Jarul & Lagerstroemia speciosa (L.) Pers. & Lythraceae & Tree & $\mathrm{LC}$ & 20172752 \\
\hline 245. & Mehedi, Hena & Lawsonia inermis L. & Lythraceae & Shrub & $\mathrm{LC}$ & 20172756 \\
\hline 246. & Dhatriphul & Woodfordia fruticosa (L.) Kurz & Lythraceae & Shrub & NT & 20172941 \\
\hline 247. & Champa & Michelia champaca $\mathrm{L}$. & Magnoliaceae & Tree & $\mathrm{LC}$ & 20172785 \\
\hline 248. & Dherosh & Abelmoschus esculentus (L.) Moench & Malvaceae & Herb & $\mathrm{LC}$ & 20172501 \\
\hline 249. & Mushakdana & Abelmoschus moschatus Medik. & Malvaceae & Shrub & $\mathrm{NE}$ & 20172502 \\
\hline 250. & Joba & Hibiscus rosa-sinensis L. & Malvaceae & Shrub & $\mathrm{LC}$ & 20172719 \\
\hline 251. & Chukhai & Hibiscus sabdariffa L. var. Sabdariffa & Malvaceae & Herb & $\mathrm{LC}$ & 20172720 \\
\hline 252. & Achar gota & Microcos paniculata $\mathrm{L}$. & Malvaceae & Shrub & $\mathrm{LC}$ & 20172786 \\
\hline 253. & Ban methi & Sida acuta Burm. f. & Malvaceae & Herb & $\mathrm{LC}$ & 20172881 \\
\hline 254. & Lal barela & Sida rhombifolia $\mathrm{L}$. & Malvaceae & Herb & $\mathrm{LC}$ & 20172882 \\
\hline 255. & Banokra & Urena lobata L. & Malvaceae & Shrub & $\mathrm{LC}$ & 20172935 \\
\hline 256. & Arraroot & Maranta arundinacea $\mathrm{L}$. & Marantaceae & Herb & $\mathrm{LC}$ & 20172779 \\
\hline 257. & Not Known & Phrynium pubinerve Blume & Marantaceae & Herb & $\mathrm{NE}$ & 20172833 \\
\hline 258. & Datranga & Melastoma malabathricum L. & Melastomataceae & Shrub & $\mathrm{LC}$ & 20172781 \\
\hline 259. & Gaichi & $\begin{array}{l}\text { Osbeckia stellata Buch.-Hum. ex Ker } \\
\text { Gawl }\end{array}$ & Melastomataceae & Shrub & $\mathrm{NE}$ & 20172815 \\
\hline 260. & Bristletips & $\begin{array}{c}\text { Oxyspora paniculata (D. Don) DC } \\
\text { var. vagans (Roxb.) }\end{array}$ & Melastomataceae & Shrub & $\mathrm{DD}$ & 20172818 \\
\hline
\end{tabular}




\begin{tabular}{|c|c|c|c|c|c|c|}
\hline $\begin{array}{c}\text { Sl } \\
\text { No } \\
\end{array}$ & Local Name & Botanical Name & Family & Habit & CS & $\begin{array}{c}\text { Specimen } \\
\text { No } \\
\end{array}$ \\
\hline 261. & Pitraj, Royna & $\begin{array}{l}\text { Aphanamixis polystachya } \\
\text { (Wall.)R.N.Parker }\end{array}$ & Meliaceae & Tree & $\mathrm{LC}$ & 20172544 \\
\hline 262. & Lal pitraj & $\begin{array}{c}\text { Chisocheton cumingianus (C.DC.) } \\
\text { Harms. }\end{array}$ & Meliaceae & Tree & $\mathrm{LC}$ & 20172596 \\
\hline 263. & Chirkrassi & Chukrasia tabularis A. Juss & meliaceae & Tree & $\mathrm{LC}$ & 20172598 \\
\hline 264. & Neem & Melia azederach $\mathrm{L}$. & Meliaceae & Tree & $\mathrm{LC}$ & 20172782 \\
\hline 265. & Mahagoni & Swietenia mahagoni (L.) Jacq. & Meliaceae & Tree & $\mathrm{LC}$ & 20172902 \\
\hline 266. & Rongi, Toon & Toona ciliata Roemer & Meliaceae & Tree & $\mathrm{LC}$ & 20172927 \\
\hline 267. & Patal pur & Cyclea barbata Miers. & Menispermaceae & Climber & NT & 20172643 \\
\hline 268. & Goria loti & Pericampylus glaucus (Lam.) Merr. & Menispermaceae & Climber & $\mathrm{LC}$ & 20172829 \\
\hline 269. & tanda manik & Stephania glabra (Roxb.) Miers & Menispermaceae & Climber & $\mathrm{LC}$ & 20172893 \\
\hline 270. & Akanadi & Stephania japonica (Thumb.) Miers & Menispermaceae & Climber & $\mathrm{LC}$ & 20172894 \\
\hline 271. & Gulancho & Tinospora cordifolia (Willd.) Miers & Menispermaceae & Climber & $\mathrm{LC}$ & 20172925 \\
\hline 272. & Gulancha & $\begin{array}{c}\text { Tinospora cripsa (L.) Hook. f. \& } \\
\text { Thomson. }\end{array}$ & Menispermaceae & Climber & NT & 20172926 \\
\hline 273. & Khair & Acacia catechu (L. f.) Willd & Mimosacea & Tree & $\mathrm{LC}$ & 20172505 \\
\hline 274. & Lajjabati & Mimusa pudica $\mathrm{L}$. & Mimosacea & Herb & $\mathrm{LC}$ & 20172791 \\
\hline 275. & Lohakat & $\begin{array}{l}\text { Xylia xylocarpa (Roxb.) Taub. Var } \\
\text { kerrii (Craib \& Hutch.) I.C. Neilsen }\end{array}$ & Mimosaceae & Tree & $\mathrm{LC}$ & 20172945 \\
\hline 276. & $\begin{array}{l}\text { Chapalish, } \\
\text { cham }\end{array}$ & $\begin{array}{c}\text { Artocarpus chama Buch-Ham. ex } \\
\text { Wall }\end{array}$ & Moraceae & Tree & $\mathrm{NE}$ & 20172552 \\
\hline 277. & Kanthal & Artocarpus heterophyllus Lamk. & Moraceae & Tree & $\mathrm{LC}$ & 20172553 \\
\hline 278. & Dewa, Borta & Artocarpus lacucha Buch.-Ham. & Moraceae & Tree & $\mathrm{LC}$ & 20172554 \\
\hline 279. & Pahari sheora & Balanostreblus ilicifolius Kurz. & Moraceae & Tree & $\mathrm{LC}$ & 20172558 \\
\hline 280. & Bot & Ficus benghalensis L. & Moraceae & Tree & $\mathrm{LC}$ & 20172686 \\
\hline 281. & Jiri bot, Pakur & Ficus benjamina L. & Moraceae & Tree & $\mathrm{LC}$ & 20172687 \\
\hline 282. & Kak dumur & Ficus hispida L. F. & Moraceae & Tree & $\mathrm{LC}$ & 20172688 \\
\hline 283. & Latalo bot & Ficus pumila L. & Moraceae & Climber & $\mathrm{LC}$ & 20172689 \\
\hline 284. & Jagya Dumur & Ficus racemosa $\mathrm{L}$. & Moraceae & Tree & $\mathrm{LC}$ & 20172690 \\
\hline 285. & Dumur & Ficus roxburghii Wall. & Moraceae & Tree & $\mathrm{LC}$ & 20172691 \\
\hline 286. & Lata dumur & Ficus scandens Roxb. & Moraceae & Climber & $\mathrm{LC}$ & 20172692 \\
\hline 287. & Sheora & Streblus asper Lour. & Moraceae & Tree & $\mathrm{LC}$ & 20172900 \\
\hline 288. & Shajina & Moringa oleifera Lam. & Moringaceae & Tree & $\mathrm{LC}$ & 20172797 \\
\hline 289. & Not Known & Horsfieldia kingii (Hook. f.) Warb. & Myristicaceae & Tree & VU & 20172727 \\
\hline 290. & Am Berela & Myristica linifolia Roxb. & Myristicaceae & Tree & $\mathrm{LC}$ & 20172803 \\
\hline 291. & Banjam & Ardisia solanacea (Poir.) Roxb. & Myrsinaceae & Shrub & NT & 20172548 \\
\hline 292. & Ramjani & Maesa indica (Roxb.) DC & Myrsinaceae & Shrub & $\mathrm{LC}$ & 20172773 \\
\hline 293. & Moricha & Maesa ramentacea (Roxb.) A. DC. & Myrsinaceae & Shrub & $\mathrm{LC}$ & 20172774 \\
\hline 294. & Peyara & Psidium guajava $\mathrm{L}$. & Myrtaceae & Tree & $\mathrm{LC}$ & 20172852 \\
\hline 295. & Kalajam, Jam & Syzygium cumini (L.) Skeeels & Myrtaceae & Tree & $\mathrm{LC}$ & 20172904 \\
\hline 296. & $\begin{array}{l}\text { Putijam, } \\
\text { kakjam }\end{array}$ & Syzygium fruticosum (Roxb.) DC. & Myrtaceae & Tree & $\mathrm{LC}$ & 20172905 \\
\hline 297. & Dhakijam & Syzygium grande (Wight) Walp. & Myrtaceae & Tree & $\mathrm{LC}$ & 20172906 \\
\hline 298. & Golap jam & Syzygium jambos (L.) Alston & Myrtaceae & Tree & $\mathrm{LC}$ & 20172907 \\
\hline 299. & Jamrul & Syzygium malaccense (L.) Merr. & Myrtaceae & Tree & $\mathrm{LC}$ & 20172908 \\
\hline 300. & Purnanova & Boerhavia diffusa $\mathrm{L}$. & Nyctaginaceae & Herb & $\mathrm{LC}$ & 20172570 \\
\hline 301. & $\begin{array}{l}\text { Banmallika, } \\
\text { Beli }\end{array}$ & Jasminum sambac (L.) Aiton & Oleaceae & Shrub & $\mathrm{LC}$ & 20172740 \\
\hline 302. & Jangli jui & Jasminum scandens Vahl & Oleaceae & Shrub & $\mathrm{LC}$ & 20172741 \\
\hline 303. & Chikna bizi & Myxopyrum smilacifolia Blume & Oleaceae & Shrub & NT & 20172804 \\
\hline 304. & Panilong & Ludwigia hyssopifolia (G.Don) Exell & Onagraceae & Herb & $\mathrm{LC}$ & 20172770 \\
\hline 305. & Not Known & Crepidium biauritum (Lindl.) D.L. & Orchidaceae & Herb & $\mathrm{EN}$ & 20172631 \\
\hline 306. & Tosabak & Cymbidium aloifolium (L.) Sw. & Orchidaceae & Epiphytes & $\mathrm{LC}$ & 20172644 \\
\hline 307. & Shankhamul & Geodorum densiflorum (Lam.) Schltr & Orchidaceae & Herb & $\mathrm{NE}$ & 20172702 \\
\hline 308. & $\begin{array}{l}\text { Patagona } \\
\text { Shiral }\end{array}$ & Nervilia aragoana Geudich. & Orchidaceae & Herb & VU & 20172805 \\
\hline 309. & Folidota & Pholidota imbricata Lindl. & Orchidaceae & Epiphytes & NT & 20172832 \\
\hline 310. & $\begin{array}{c}\text { Shial leza } \\
\text { orchid }\end{array}$ & Rhynchostylis retusa (L.) Blume & Orchidaceae & Epiphytes & $\mathrm{LC}$ & 20172863 \\
\hline
\end{tabular}




\begin{tabular}{|c|c|c|c|c|c|c|}
\hline $\begin{array}{r}\text { Sl } \\
\text { No }\end{array}$ & Local Name & Botanical Name & Family & Habit & $\mathbf{C S}$ & $\begin{array}{c}\text { Specimen } \\
\text { No }\end{array}$ \\
\hline 311. & Bilimbi & Averrhoa bilimbi $\mathrm{L}$. & Oxalidaceae & Tree & $\mathrm{LC}$ & 20172555 \\
\hline 312. & Kamranga & Averrhoa carambola $\mathrm{L}$. & Oxalidaceae & Tree & $\mathrm{LC}$ & 20172556 \\
\hline 313. & Amrul shak & Oxalis corniculata $\mathrm{L}$. & Oxalidaceae & Herb & $\mathrm{LC}$ & 20172817 \\
\hline 314. & $\begin{array}{l}\text { Chata Pat, } \\
\text { Kurud }\end{array}$ & Licuala peltata Roxb. & Palmae & Tree & VU & 20172764 \\
\hline 315. & $\begin{array}{l}\text { Popy, } \\
\text { Shialkanta }\end{array}$ & Argemone maxicana $\mathrm{L}$. & Papaveraceae & Herb & $\mathrm{LC}$ & 20172549 \\
\hline 316. & Jhumkolata & Passiflora foetida $\mathrm{L}$. & Passifloraceae & Climber & $\mathrm{LC}$ & 20172822 \\
\hline 317. & Lotkon & Baccaurea ramiflora Lour & Phyllantanceae & Tree & $\mathrm{LC}$ & 20172557 \\
\hline 318. & Khudijam & Antidesma ghaesembilla Gaertn. & Phyllanthaceae & Tree & $\mathrm{LC}$ & 20172543 \\
\hline 319. & Kokra & Aporosa wallichii Hook. f. & Phyllanthaceae & Tree & $\mathrm{NE}$ & 20172546 \\
\hline 320. & Kanjol Bhadi & Bischofia javanica Blume. & Phyllanthaceae & Tree & $\mathrm{LC}$ & 20172566 \\
\hline 321. & Amloki & Emblica officinalis Gaertn. & Phyllanthaceae & Tree & $\mathrm{LC}$ & 20172679 \\
\hline 322. & Vuiamla & Phyllanthus niruri L. & Phyllanthaceae & Herb & $\mathrm{LC}$ & 20172834 \\
\hline 323. & Orboroi & Phyllunthus acidus (L.) Skeels & Phyllanthaceae & Tree & $\mathrm{LC}$ & 20172835 \\
\hline 324. & Not Known & Peperomia caperata Yunck. & Piperaceae & Herb & $\mathrm{LC}$ & 20172827 \\
\hline 325. & Luchi Pata & Peperomia pellucida (L.) Kunth. & Piperaceae & Herb & $\mathrm{LC}$ & 20172828 \\
\hline 326. & Chui jhal & Piper chaba Hunter & Piperaceae & Climber & $\mathrm{LC}$ & 20172838 \\
\hline 327. & Pipla & Piper longum L. & Piperaceae & Climber & $\mathrm{LC}$ & 20172839 \\
\hline 328. & Ban pipul & Piper rhytidocarpum Hook. f. & Piperaceae & Climber & $\mathrm{LC}$ & 20172840 \\
\hline 329. & Pahari pipul & Piper sylvaticum Roxb. & Piperaceae & Climber & NT & 20172841 \\
\hline 330. & Lemon grass & $\begin{array}{c}\text { Cymbopogon citratus (DC. ex Nees) } \\
\text { Stapf }\end{array}$ & Poaceae & Herb & NT & 20172645 \\
\hline 331. & Makra & Dactyloctenium aegyptium (L.) Willd. & Poaceae & Herb & $\mathrm{LC}$ & 20172648 \\
\hline 332. & Renga grass & Saccharum arundinaceum Retc. & Poaceae & Herb & $\mathrm{LC}$ & 20172868 \\
\hline 333. & Udodhan & Setaria palmifolia (J.König) Stapf & Poaceae & Herb & $\mathrm{LC}$ & 20172880 \\
\hline 334. & $\begin{array}{c}\text { Kaker } \\
\text { Pantabhat }\end{array}$ & Ampelygonum chinense (L.) Lindl. & Polygonaceae & Climber & $\mathrm{LC}$ & 20172535 \\
\hline 335. & Bish katali & $\begin{array}{c}\text { Persicaria acuminata (Kunth) } \\
\text { M.Gómez }\end{array}$ & Polygonaceae & Herb & $\mathrm{LC}$ & 20172831 \\
\hline 336. & Pani marich & Presicaria hydropiper L. & Polygonaceae & Herb & $\mathrm{LC}$ & 20172849 \\
\hline 337. & Nunia shak & Portulaca oleracea $\mathrm{L}$. & Portulacaceae & Herb & $\mathrm{LC}$ & 20172843 \\
\hline 338. & Kachuripana & Eichhornia crassipes (Mart.) Solms & Potenderiaceae & Herb & $\mathrm{LC}$ & 20172676 \\
\hline 339. & Dalim & Punica granatum L. & Punicaceae & Tree & $\mathrm{LC}$ & 20172857 \\
\hline 340. & Ban boroi & Zizyphus oenoplia (L.) Mill & Rhamnaceae & Shrub & $\mathrm{LC}$ & 20172951 \\
\hline 341. & Matang & Carallia brachiata (Lour.) Merr. & Rhizophoraceae & Tree & $\mathrm{LC}$ & 20172581 \\
\hline 342. & Ceylon cherry & Prunus ceylanica (Wight) Miq. & Rosaceae & Tree & $\mathrm{NE}$ & 20172851 \\
\hline 343. & Lalmalakka & Rubus moluccanus L. & Rosaceae & Climber & $\mathrm{LC}$ & 20172867 \\
\hline 344. & Haldu & Adina cordifolia (Roxb.) Ridsdale & Rubiaceae & Tree & NT & 20172512 \\
\hline 345. & Kadam & Anthocephalus cadamba (Roxb.) Miq. & Rubiaceae & Tree & $\mathrm{LC}$ & 20172542 \\
\hline 346. & Botta & Gardenia coronaria Buch. Ham. & Rubiaceae & Tree & $\mathrm{LC}$ & 20172700 \\
\hline 347. & Not Known & Hedyotis thomsonii Hook. f. & Rubiaceae & Herb & $\mathrm{EN}$ & 20172715 \\
\hline 348. & Not Known & $\begin{array}{l}\text { Hedyotis verticillata (Linnaeus) } \\
\text { Lamarck }\end{array}$ & Rubiaceae & Herb & $\mathrm{LC}$ & 20172716 \\
\hline 349. & Java rangan & Ixora javanica (Blume) DC. & Rubiaceae & Shrub & $\mathrm{LC}$ & 20172736 \\
\hline 350. & Shada rangan & Ixora lanceolata Lam. & Rubiaceae & Shrub & $\mathrm{LC}$ & 20172737 \\
\hline 351. & Kuthi rangan & $\begin{array}{c}\text { Ixora nigricans } \mathrm{R} \text {. Br. ex Wight \& } \\
\text { Arn. }\end{array}$ & Rubiaceae & Shrub & NT & 20172738 \\
\hline 352. & Shum rangan & Ixora spectabilis Wall. Ex G. Don & Rubiaceae & Shrub & VU & 20172739 \\
\hline 353. & Nagaballi & Mussaenda frondosa $\mathrm{L}$. & Rubiaceae & Shrub & $\mathrm{LC}$ & 20172799 \\
\hline 354. & Mussaenda & Mussaenda roxburghii Hook.f. & Rubiaceae & Shrub & NT & 20172800 \\
\hline 355. & Mycetia & Mycetia longifolia (Wall.) Kuntz. & Rubiaceae & Shrub & VU & 20172801 \\
\hline 356. & Khetpapri & Oldenlandia corymbosa $\mathrm{L}$. & Rubiaceae & Herb & $\mathrm{LC}$ & 20172809 \\
\hline 357. & Harigandhali & Ophiorrhiza harissiana B. Heyne & Rubiaceae & Herb & $\mathrm{NE}$ & 20172810 \\
\hline 358. & Pislagandhali & Ophiorrhiza villosa Roxb. & Rubiaceae & Herb & VU & 20172811 \\
\hline 359. & Gandhabhaduli & Paedaria foetida $\mathrm{L}$. & Rubiaceae & Climber & $\mathrm{LC}$ & 20172819 \\
\hline 360. & Bana mali & Pavetta indica L. var. indica & Rubiaceae & Shrub & $\mathrm{LC}$ & 20172823 \\
\hline 361. & Lal bhuta & Psychotria calocarpa Kurz. & Rubiaceae & Shrub & NT & 20172854 \\
\hline 362. & Cat's claw & Uncaria tomentosa (Willd. ex. & Rubiaceae & Climber & NT & 20172932 \\
\hline
\end{tabular}




\begin{tabular}{|c|c|c|c|c|c|c|}
\hline $\begin{array}{c}\text { Sl } \\
\text { No }\end{array}$ & Local Name & Botanical Name & Family & Habit & CS & $\begin{array}{c}\text { Specimen } \\
\text { No }\end{array}$ \\
\hline & & Schult.) DC. & & & & \\
\hline 363. & Banjamir & Acronychia pedunculata (L.) Miq. & Rutaceae & Tree & $\mathrm{NE}$ & 20172509 \\
\hline 364. & Bel & Aegle marmelos (L.) Corr. & Rutaceae & Tree & $\mathrm{LC}$ & 20172513 \\
\hline 365. & Lebu & Citrus aurantiifolia (Christm.) Suingle & Rutaceae & Shrub & $\mathrm{LC}$ & 20172608 \\
\hline 366. & Rough lemon & Citrus jambhiri Lush. & Rutaceae & Tree & $\mathrm{LC}$ & 20172609 \\
\hline 367. & Jambura & Citrus maxima (Burm.f.) Merr. & Rutaceae & Tree & $\mathrm{LC}$ & 20172610 \\
\hline 368. & Pan karpur & Clasusena excavate Burm.f. & Rutaceae & Tree & $\mathrm{LC}$ & 20172611 \\
\hline 369. & $\begin{array}{l}\text { Moricha, Pan } \\
\text { mauri }\end{array}$ & $\begin{array}{c}\text { Clausena heptaphylla (Roxb.) Wight } \\
\& \text { Arn. }\end{array}$ & Rutaceae & Tree & $\mathrm{LC}$ & 20172612 \\
\hline 370. & Datnajan & Glycosmis pentaphylla (Retz) & Rutaceae & Shrub & $\mathrm{LC}$ & 20172706 \\
\hline 371. & Bankunch & $\begin{array}{c}\text { Micromelum minutum (G.Forst.) } \\
\text { Wight \& Arn. }\end{array}$ & Rutaceae & Tree & $\mathrm{LC}$ & 20172787 \\
\hline 372. & Currypata & Murraya koenigii (L.) Spreng & Rutaceae & Shrub & $\mathrm{LC}$ & 20172798 \\
\hline 373. & Katachita & Allophylus serratus (Roxb.) Prain & Sapindaceae & Tree & $\mathrm{NE}$ & 20172518 \\
\hline 374. & phutka & Cardiospermum halicacabum L. & Sapindaceae & Herb & $\mathrm{LC}$ & 20172582 \\
\hline 375. & Puli pita gach & Harpullia arborea (Blanco) Radlk. & Sapindaceae & Shrub & VU & 20172712 \\
\hline 376. & Litchu & Litchi chinensis Sonn. & Sapindaceae & Tree & $\mathrm{LC}$ & 20172767 \\
\hline 377. & Bura & Xerospermum laevigatum Radlk. & Sapindaceae & Tree & VU & 20172944 \\
\hline 378. & Sofeda & Achras zapota L. & Sapotaceae & Tree & $\mathrm{LC}$ & 20172507 \\
\hline 379. & Lali & $\begin{array}{c}\text { Palaquium polyanthum (Wall. ex G. } \\
\text { Don.) Baill }\end{array}$ & Sapotaceae & Tree & $\mathrm{NE}$ & 20172821 \\
\hline 380. & Kadsura & Kadsura heteroclita (Roxb.) Craib & Schisandraceae & Climber & $\mathrm{NE}$ & 20172745 \\
\hline 381. & Kumarilata & Smilax prolifera Roxb & Smilaceae & Climber & NT & 20172883 \\
\hline 382. & Datura & Datura metel L. & Solanaceae & Herb & $\mathrm{LC}$ & 20172649 \\
\hline 383. & Phutka & Physalis micrantha Link. & Solanaceae & Herb & $\mathrm{LC}$ & 20172836 \\
\hline 384. & Katabegun & Solanum sisymbrifolium Lam. & Solanaceae & Shrub & $\mathrm{LC}$ & 20172884 \\
\hline 385. & Jangli begum & Solanum torvum Sw. & Solanaceae & Shrub & $\mathrm{LC}$ & 20172885 \\
\hline 386. & Bandorhola & $\begin{array}{c}\text { Duabanga grandiflora (Roxb. ex DC.) } \\
\text { Walp. }\end{array}$ & Sonneratiaceae & Tree & $\mathrm{LC}$ & 20172674 \\
\hline 387. & Lalgurania alu & Stemona tuberosa Lour. & Stemonaceae & Climber & NT & 20172892 \\
\hline 388. & Ulatkambal & Abroma augusta L. & Sterculiaceae & Shrub & NT & 20172504 \\
\hline 389. & Harjora & Byttneria pilosa Roxb. & Sterculiaceae & Climber & $\mathrm{LC}$ & 20172574 \\
\hline 390. & $\begin{array}{l}\text { Buddha- } \\
\text { Narike }\end{array}$ & Pterygota alata (Roxb.) R.Br. & Sterculiaceae & Tree & $\mathrm{LC}$ & 20172855 \\
\hline 391. & Shampan & $\begin{array}{l}\text { Scaphium scaphigerum (Wall. ex } \\
\text { G.Don) Guibourt \& Planch. }\end{array}$ & Sterculiaceae & Tree & VU & 20172872 \\
\hline 392. & Toni udal & Sterculia hamiltonii (Kuntze) Adelb & Sterculiaceae & Shrub & $\mathrm{LC}$ & 20172895 \\
\hline 393. & Udal & Sterculia villosa Roxb. ex DC. & Sterculiaceae & Tree & $\mathrm{LC}$ & 20172896 \\
\hline 394. & Borobhauri & $\begin{array}{l}\text { Symplocos macrophylla Wall. ex DC } \\
\text { ssp. Macrophylla }\end{array}$ & Symplocaceae & Tree & VU & 20172903 \\
\hline 395. & Badurfuli & Tacca chantrieri Andre & Taccaceae & Shrub & $\mathrm{LC}$ & 20172911 \\
\hline 396. & Bara hikond & Tacca integrifolia Ker Gawl. & Taccaceae & Shrub & $\mathrm{NE}$ & 20172912 \\
\hline 397. & Chundul & Tetrameles nudiflora $\mathrm{R} . \mathrm{Br}$. & Tetramelaceae & Tree & $\mathrm{NE}$ & 20172922 \\
\hline 398. & Agor & Aquilaria agallocha Lamk. & Thymeleaceae & Tree & $\mathrm{LC}$ & 20172547 \\
\hline 399. & Titpata & Corchorus aestuans L. & Tiliaceae & Shrub & $\mathrm{LC}$ & 20172626 \\
\hline 400. & Panicherra & Grewia serrulata DC. & Tiliaceae & Shrub & $\mathrm{LC}$ & 20172709 \\
\hline 401. & Jigni shuktani & Trema orientalis (L.) Blume & Ulmaceae & Tree & $\mathrm{LC}$ & 20172928 \\
\hline 402. & Malabaruli & Boehmaria malabarica Wedd. & Urticaceae & Shrub & $\mathrm{LC}$ & 20172569 \\
\hline 403. & Silajhara & Elatostema papillosum wedd. & Urticaceae & Herb & $\mathrm{NE}$ & 20172678 \\
\hline 404. & Lal bichuti & Fleurya interrupta (L.) Gaudich & Urticaceae & Herb & $\mathrm{LC}$ & 20172697 \\
\hline 405. & Not Known & Laportea crenulata Gaud. & Urticaceae & Shrub & $\mathrm{LC}$ & 20172754 \\
\hline 406. & Not Known & Pellionia heyneana Weddell. & Urticaceae & Herb & $\mathrm{NE}$ & 20172826 \\
\hline 407. & Pilea & Pilea melastomoides (Poir.) Wedd. & Urticaceae & Shrub & $\mathrm{EN}$ & 20172837 \\
\hline 408. & Hirazolzi & Pouzolzia hirta (Blume) Hassk. & Urticaceae & Herb & $\mathrm{LC}$ & 20172845 \\
\hline 409. & Check & Pouzolzia indica (L.) Gaudich & Urticaceae & Herb & $\mathrm{LC}$ & 20172846 \\
\hline 410. & Jangallya Shak & Sarcochlamys pulcherrima Guadich. & Urticaceae & Shrub & $\mathrm{NE}$ & 20172870 \\
\hline 411. & Bormala & Callicarpa arborea (Roxb.) & Verbenaceae & Tree & $\mathrm{LC}$ & 20172577 \\
\hline 412. & Bamun hati & Clerodendrum indicum L. Kuntze. & Verbenaceae & Shrub & $\mathrm{LC}$ & 20172614 \\
\hline 413. & Rakta bhait & Clerodendrum japonicum (Thunb.) & Verbenaceae & Shrub & NT & 20172615 \\
\hline
\end{tabular}




\begin{tabular}{|c|c|c|c|c|c|c|}
\hline $\begin{array}{c}\text { Sl } \\
\text { No }\end{array}$ & Local Name & Botanical Name & Family & Habit & CS & $\begin{array}{c}\text { Specimen } \\
\text { No }\end{array}$ \\
\hline & & Sweet & & & & \\
\hline 414. & Bhait & Clerodendrum viscosum Vent. & Verbenaceae & Shrub & $\mathrm{LC}$ & 20172616 \\
\hline 415. & Gamar & Gmelina arborea Roxb. & Verbenaceae & Tree & $\mathrm{LC}$ & 20172707 \\
\hline 416. & Lantana & Lantana camera $\mathrm{L}$. & Verbenaceae & Shrub & $\mathrm{LC}$ & 20172753 \\
\hline 417. & Lalang & Premna esculenta Roxb. & Verbenaceae & Shrub & $\mathrm{LC}$ & 20172847 \\
\hline 418. & Goniari & Premna integrifolia $\mathrm{L}$. & Verbenaceae & Shrub & $\mathrm{NE}$ & 20172848 \\
\hline 419. & Segun & Tectona grandis L.f. & Verbenaceae & Tree & $\mathrm{LC}$ & 20172915 \\
\hline 420. & Horina & Vitex peduncularis Wall. ex Schauer & Verbenaceae & Tree & $\mathrm{NE}$ & 20172937 \\
\hline 421. & $\begin{array}{c}\text { Japani } \\
\text { goalilata }\end{array}$ & Cayratia japonica (Thunb.) Gagnep & Vitaceae & Climber & $\mathrm{LC}$ & 20172590 \\
\hline 422. & Goalilata & Cayratia pedata (Lam.) Gagnep. & Vitaceae & Climber & NT & 20172591 \\
\hline 423. & Not Known & $\begin{array}{c}\text { Cayratia tenuifolia (Wight \& Arn.) } \\
\text { Gagnep }\end{array}$ & Vitaceae & Climber & NT & 20172592 \\
\hline 424. & Alianga-lata & Cissus adnata Roxb. & Vitaceae & Climber & $\mathrm{LC}$ & 20172602 \\
\hline 425. & Amashalata & Cissus assamica (Lawson) Craib & Vitaceae & Climber & $\mathrm{LC}$ & 20172603 \\
\hline 426. & Begonia lata & Cissus discolor Blume & Vitaceae & Climber & $\mathrm{LC}$ & 20172604 \\
\hline 427. & Rangila lata & Cissus javanica DC. & Vitaceae & Climber & $\mathrm{LC}$ & 20172605 \\
\hline 428. & Tok patha & Cissus repens Lam. & Vitaceae & Climber & DD & 20172606 \\
\hline 429. & Agrorida & Cissus repanda (Wight \& Arn.) Vahl & Vitaceae & Climber & $\mathrm{LC}$ & 20172607 \\
\hline 430. & Not Known & Vitis flexuosa Thunb. & Vitaceae & Climber & $\mathrm{NE}$ & 20172938 \\
\hline 431. & Konchi elachi & Alpinia conchigera Griff. & Zingiberaceae & Herb & $\mathrm{LC}$ & 20172524 \\
\hline 432. & Deo tara & Alpinia malaccensis Brum.f. & Zingiberaceae & Herb & $\mathrm{LC}$ & 20172525 \\
\hline 433. & Not Known & Alpinia mutica Roscoe & Zingiberaceae & Herb & $\mathrm{NE}$ & 20172526 \\
\hline 434. & Tara & Alpinia nigra (Gaertn.) Burtt & Zingiberaceae & Herb & $\mathrm{LC}$ & 20172527 \\
\hline 435. & Kalo halud & Curcuma caesia Roxb. & Zingiberaceae & Herb & $\mathrm{LC}$ & 20172640 \\
\hline 436. & Halud & Curcuma longa $\mathrm{L}$. & Zingiberaceae & Herb & $\mathrm{LC}$ & 20172641 \\
\hline 437. & Shoti & Curcuma zedoaria (Christm.) Roscoe & Zingiberaceae & Herb & $\mathrm{LC}$ & 20172642 \\
\hline 438. & Jhuli globba & Globba pendula Roxb. & Zingiberaceae & Herb & EN & 20172704 \\
\hline 439. & Vui ada & $\begin{array}{l}\text { Hedychium coccineum Buch.-hum ex } \\
\text { Sm. }\end{array}$ & Zingiberaceae & Herb & $\mathrm{LC}$ & 20172713 \\
\hline 440. & Not Known & Hedychium gardnerianum Roscoe & Zingiberaceae & Herb & $\mathrm{NE}$ & 20172714 \\
\hline 441. & Sichnana & Hitchenia careyana Benth. & Zingiberaceae & Herb & $\mathrm{NE}$ & 20172721 \\
\hline 442. & Chandumula & Kaempferia galanga L. & Zingiberaceae & Herb & $\mathrm{LC}$ & 20172746 \\
\hline 443. & Vui chapa & Kaempferia rotunda $\mathrm{L}$. & Zingiberaceae & Herb & $\mathrm{LC}$ & 20172747 \\
\hline 444. & Kala bach & $\begin{array}{c}\text { Kamepferia parvifolara Wall. Ex } \\
\text { Baker }\end{array}$ & Zingiberaceae & Herb & $\mathrm{LC}$ & 20172748 \\
\hline 445. & Dancing Girl & Mantisia spathulata (Roxb.) Schult. & Zingiberaceae & Herb & $\mathrm{LC}$ & 20172778 \\
\hline 446. & Jongli ada & Zingiber capitatum Roxb. & Zingiberaceae & Herb & $\mathrm{NE}$ & 20172946 \\
\hline 447. & Ban Ada & $\begin{array}{c}\text { Zingiber montanum (J.König) Link ex } \\
\text { A.Dietr. }\end{array}$ & Zingiberaceae & Herb & $\mathrm{LC}$ & 20172947 \\
\hline 448. & Ada & Zingiber officinale Rosc. & Zingiberaceae & Herb & $\mathrm{LC}$ & 20172948 \\
\hline 449. & Murgagachh & Zingiber rubens Roxb. & Zingiberaceae & Herb & NT & 20172949 \\
\hline 450. & Mahabaribach & Zingiber zerumbet (L.) Roscoe ex Sm. & Zingiberaceae & Herb & $\mathrm{LC}$ & 20172950 \\
\hline
\end{tabular}

CS=Conservation Status; LC=Least concern; NT=Near threatened; VU=Vulnearable; $\mathrm{EN}=$ Endangered; $\mathrm{DD}=$ Data Deficient; NE=Not evaluated

(C) 2020 by the authors. Submitted for possible open access publication under the terms and conditions of the Creative Commons Attribution (CC BY) license (http://creativecommons.org/licenses/by/4.0/). 